\title{
Review \\ Crown-Like Structures in Breast Adipose Tissue: Early Evidence and Current Issues in Breast Cancer
}

\author{
Maret L. Maliniak ${ }^{1, * \mathbb{D}}$, Jasmine Miller-Kleinhenz ${ }^{1}$, Deirdre P. Cronin-Fenton ${ }^{2}$, Timothy L. Lash ${ }^{1,2,3} \mathbb{D}$, \\ Keerthi Gogineni ${ }^{3,4}$, Emiel A. M. Janssen ${ }^{5}$ iD and Lauren E. McCullough ${ }^{1,3}$ (D)
}

1 Department of Epidemiology, Rollins School of Public Health, Emory University, Atlanta, GA 30322, USA; jmill37@emory.edu (J.M.-K.); timothy.lee.lash@emory.edu (T.L.L.); lauren.mccullough@emory.edu (L.E.M.)

2 Department of Clinical Epidemiology, Aarhus University Hospital, 8200 Aarhus, Denmark; dc@clin.au.dk

3 Glenn Family Breast Center, Winship Cancer Institute of Emory University, Atlanta, GA 30322, USA; keerthi.gogineni@emory.edu

4 Department of Hematology and Medical Oncology, Emory University School of Medicine, Atlanta, GA 30322, USA

5 Department of Pathology, Stavanger University Hospital, 4011 Stavanger, Norway; emilius.adrianus.maria.janssen@sus.no

* Correspondence: mmalini@emory.edu

check for

updates

Citation: Maliniak, M.L.; Miller-Kleinhenz, J.; Cronin-Fenton, D.P.; Lash, T.L.; Gogineni, K.; Janssen, E.A.M.; McCullough, L.E.

Crown-Like Structures in Breast Adipose Tissue: Early Evidence and Current Issues in Breast Cancer. Cancers 2021, 13, 2222. https:// doi.org/10.3390/cancers13092222

Academic Editor: Marco Falasca

Received: 9 March 2021

Accepted: 26 April 2021

Published: 6 May 2021

Publisher's Note: MDPI stays neutral with regard to jurisdictional claims in published maps and institutional affiliations.

Copyright: (c) 2021 by the authors. Licensee MDPI, Basel, Switzerland. This article is an open access article distributed under the terms and conditions of the Creative Commons Attribution (CC BY) license (https:// creativecommons.org/licenses/by/ $4.0 /)$.
Simple Summary: Obesity increases the risk of postmenopausal, hormone receptor-positive breast cancer and has been linked to a higher risk of recurrence and mortality. During obesity, adipose tissue can become dysfunctional, resulting in chronic low-grade inflammation. Crown-like structures in breast adipose tissue (CLS-B), composed of macrophages surrounding dead or dying adipocytes in a crown-like pattern, are a new histologic marker of local inflammation. In this review, we aim to evaluate the early evidence of CLS-B in breast cancer. There is consistent evidence that CLS-B are more frequently detected among obese compared to non-obese breast cancer patients. Additionally, several studies have found that CLS-B presence is associated with metabolic and inflammatory factors that contribute to breast cancer development and progression. However, more studies are needed to understand the potential clinical utility of CLS-B as a marker of breast cancer risk or prognosis.

Abstract: Obesity is an established risk factor for postmenopausal breast cancer and has been linked to worse breast cancer prognosis, most clearly for hormone receptor-positive breast cancers. The underlying mechanisms of the obesity-breast cancer association are not fully understood, but growing evidence points to the breast adipose tissue microenvironment playing an important role. Obesity-induced adipose tissue dysfunction can result in a chronic state of low-grade inflammation. Crown-like structures of the breast (CLS-B) were recently identified as a histologic marker of local inflammation. In this review, we evaluate the early evidence of CLS-B in breast cancer. Data from preclinical and clinical studies show that these inflammatory lesions within the breast are associated with local NF- $\mathrm{KB}$ activation, increased aromatase activity, and elevation of pro-inflammatory mediators (TNF $\alpha, \mathrm{IL}-1 \beta, \mathrm{IL}-6$, and COX-2-derived PGE 2 - factors involved in multiple pathways of breast cancer development and progression. There is also substantial evidence from epidemiologic studies that CLS-B are associated with greater adiposity among breast cancer patients. However, there is insufficient evidence that CLS-B impact breast cancer risk or prognosis. Comparisons across studies of prognosis were complicated by differences in CLS-B evaluation and deficiencies in study design, which future studies should take into consideration. Breast adipose tissue inflammation provides a plausible explanation for the obesity-breast cancer association, but further study is needed to establish its role and whether markers such as CLS-B are clinically useful.

Keywords: crown-like structures; obesity; inflammation; breast cancer 


\section{Introduction}

Obesity is an established risk factor for postmenopausal breast cancer [1,2] and has been linked to a higher risk of recurrence and mortality [1,3,4]. Various mechanisms have been proposed by which obesity affects breast cancer. Broadly, these include hormonal effects and chronic inflammation occurring at both the systemic and local levels [5]. Most epidemiologic studies have examined these mechanisms by measuring systemic levels of sex hormones and inflammatory markers. Less is known about the local, tissue-level effects, which are thought to be distinct and better related to the carcinogenic process [6]. Crown-like structures in the breast adipose tissue (CLS-B) are a histologic marker of local inflammation that may provide biologic insight into the obesity-breast cancer association $[7,8]$. Below, we briefly review the relation between obesity and breast cancer risk and prognosis; summarize the current epidemiological findings for the role of CLS-B in breast cancer; and conclude with possible future directions for research in this topic.

\section{The Complex Relationship between Obesity and Breast Cancer}

The association between obesity, most commonly measured using body mass index (BMI), and breast cancer risk is complex. It depends on the age that obesity is assessed, menopausal status at diagnosis, and hormone receptor (HR) status-as discussed in several reviews [9-11]. According to the most recent meta-analysis by the World Cancer Research Fund International Continuous Update Project (WCRF CUP), elevated BMI in early adulthood ( $18-30$ years) is associated with a lower risk of both premenopausal (per $5 \mathrm{~kg} / \mathrm{m}^{2}$; summary RR: $0.86,95 \% \mathrm{CI}: 0.78$ to 0.96 ) and postmenopausal breast cancer (per $5 \mathrm{~kg} / \mathrm{m}^{2}$; summary RR: $0.81,95 \% \mathrm{CI}: 0.75$ to 0.87 ) [11]. Being overweight or obese as an adult before menopause is also inversely associated with premenopausal breast cancer risk (per $5 \mathrm{~kg} / \mathrm{m}^{2}$ increase in BMI; summary RR: 0.94, 95\%CI: 0.91 to 0.98) [11] although some studies suggest that this inverse association is limited to HR-positive tumors [12-14]. There is emerging evidence of null or positive associations among premenopausal women with HR-negative tumors, particularly triple-negative breast cancers (TNBC) [14-17]. In contrast, elevated BMI in adulthood is consistently related to a higher risk of postmenopausal breast cancer-with the association limited to HR-positive tumors (per $5 \mathrm{~kg} / \mathrm{m}^{2}$; summary RR: $1.18,95 \%$ CI: 1.10 to 1.27$)$ [11].

When other adiposity measures are considered, particularly indicators of central adiposity, positive associations are observed for postmenopausal breast cancers that are generally limited to HR-positive tumors and attenuate after adjusting for BMI [18-21]. Associations differ for premenopausal breast cancers. For example, waist circumference is positively associated with premenopausal breast cancer risk when adjusting for BMI (per $10 \mathrm{~cm}$; summary RR: 1.15 , 95\%CI: 1.05 to 1.26 ) but not without BMI adjustment (per $10 \mathrm{~cm}$; summary RR: 0.99 , 95\%CI: 0.94 to 1.03) [11]. Associations for waist-to-hip ratio (WHR) similarly become positive when adjusting for BMI [11], providing compelling evidence that the localization of fat depots is important in understanding premenopausal breast cancer risk. Emerging evidence has also shown that central adiposity is more strongly associated with estrogen receptor (ER)-negative/progesterone receptor (PR)-negative tumors than ER-positive/PR-positive tumors for premenopausal breast cancers [18-20]. These results suggest that estrogen-independent mechanisms such as inflammation [22,23] and insulin signaling pathways $[24,25]$ may play a larger role for premenopausal breast cancers. Increased central adiposity is characterized by increased visceral adipose tissue, which is more metabolically active, insulin resistant, and contains more inflammatory and immune cells than subcutaneous adipose tissue [26,27].

Jiralerspong and Goodwin reviewed the role of obesity on breast cancer prognosis [1] The authors reported that obesity is associated with a 35\% to $40 \%$ increased risk of breast cancer recurrence and death regardless of menopausal or HR status [1]. This conclusion was based on three large meta-analyses of breast cancer mortality $[3,4,28]$ and five epidemiologic studies of recurrence [29-33], with positive associations observed for distant recurrence. The WCRF CUP meta-analysis estimate for breast cancer mortality, comparing the highest 
versus lowest BMI categories, is consistent with their conclusions (summary RR: 1.35, 95\%CI: 1.23 to 1.48) [34]. Although there is limited evidence of heterogeneity by HR status, meta-analyses and epidemiologic investigations show stronger and more consistently positive associations for ER-positive than ER-negative breast cancers. A recent metaanalysis of nine studies found no association between obesity and disease-free survival among triple-negative breast cancer (TNBC) patients ( $\geq 30$ versus $<30 \mathrm{~kg} / \mathrm{m}^{2}$ : summary $\mathrm{RR}=0.98,95 \% \mathrm{CI}: 0.77$ to 1.24 ) [35]. Various reasons could explain the mixed results for ER-negative cancers such as TNBC, including heterogeneity within the ER-negative and TNBC molecular subtypes or heterogeneity in study populations and methods [1]. Further research is needed to clarify associations among ER-negative patients.

The complex relationship between obesity and breast cancer points to multiple pathways by which obesity is linked to breast cancer-most thought to be risk promoting, but some of which might be risk reducing either directly or indirectly. Various mechanisms have been proposed as described in previous reviews [36-39]. To date, much of the epidemiologic evidence is limited to studies examining the systemic effects of adiposity. However, recent research is shifting towards exploring the breast adipose tissue microenvironment as a driver of carcinogenesis, which may be unique from other adipose depots [40].

\subsection{Obesity-Induced Changes in Adipose Tissue}

Obesity is characterized by adipose tissue expansion [22]. Adipose tissue was previously thought to be inert and primarily for energy storage, but it is now recognized as one of the body's largest endocrine organs, capable of secreting $>50$ adipokines, cytokines, and chemokines [22]. Adipose tissue is comprised of adipocytes but also includes preadipocytes, fibroblasts, macrophages, lymphocytes, pericytes, extracellular matrix, and endothelial cells [41]. Expansion of adipose tissue, specifically white adipose tissue (WAT), during periods of weight gain results in adipose tissue dysfunction and inflammation. The consequences of obesity-induced adipose tissue dysfunction have been summarized in a previous review by Picon-Ruiz et al. (see Figure 4 of the review by Picon-Ruiz et al.) [37]. They include increased secretion of pro-inflammatory adipokines and cytokines such as leptin, interleukin (IL)- $1 \beta$, IL-6, and tumor necrosis factor (TNF)- $\alpha$, as well as a decrease in adiponectin, an anti-inflammatory adipokine [22]. This pro-inflammatory state stimulates lipolysis and secretion of free fatty acids that can subsequently induce insulin resistance, leading to higher circulating levels of insulin and insulin-like growth factors (IGFs) [22].

Importantly for breast cancer, aromatase-the rate-limiting enzyme for estrogen biosynthesis-is stimulated in the adipose tissue by adipose-derived factors (IL-1 $\beta$, IL-6, TNF- $\alpha$, and prostaglandin (PG) $\mathrm{E}_{2}$ ) as well as liver-derived IGF-1 [22]. Moreover, increased insulin associated with overnutrition reduces hepatic production of sex hormone-binding globulin (SHBG), which binds to estradiol. This combination of increased aromatization in adipose tissue and lower SHBG to bind to estradiol results in increased estrogen production and bioavailability. After menopause, adipose tissue becomes the main source of local and circulating estrogens, the primary driver of ER-positive cancers among postmenopausal women. There is substantial epidemiologic evidence demonstrating that circulating estrogens, namely free estradiol, mediate the association between BMI and postmenopausal ER-positive breast cancer [42-44]. Furthermore, the increased risk of postmenopausal ER-positive breast cancer associated with higher BMI is not observed among women taking exogenous sex hormones who have elevated levels of circulating estrogens regardless of BMI [45].

There is also a shift in the adipose tissue immune cell landscape with obesity, leading to an increase in macrophages, particularly of the pro-inflammatory phenotype. WAT in lean individuals contains $10-15 \%$ macrophages that tend to be polarized toward the anti-inflammatory M2 phenotype, which is associated with adipose tissue metabolic homeostasis and insulin sensitivity [46]. In contrast, WAT in obese individuals contains 50-60\% macrophages that tend to be polarized toward the pro-inflammatory M1 phenotype. M1 macrophages produce pro-inflammatory cytokines that contribute to sustained adipose 
inflammation [46]. Additionally, as adipocytes undergo hypertrophy, some outgrow their blood supply, leading to hypoxia and activating hypoxia-inducible factor-1 (HIF-1). HIF-1 upregulates leptin and vascular endothelial growth factor (VEGF) and inhibits adiponectinfactors that can promote the invasion and metastasis of cancer [37]. Hypoxia can also lead to adipocyte stress and death. Macrophages surround dead or dying adipocytes in a crownlike pattern, thus presenting pathologically as crown-like structures (CLS). CLS-associated macrophages intensely produce pro-inflammatory mediators and are beginning to be recognized as a hallmark of WAT inflammation [22]. In addition to cytokine overproduction, CLS in gluteal and abdominal fat have been associated with altered adipose tissue gene expression, systemic insulin resistance, and vascular endothelial dysfunction [47,48].

Adipocytes and macrophages, such as those that form CLS, produce numerous proinflammatory factors. These factors promote metabolic dysregulation and chronic lowgrade inflammation within the adipose tissue microenvironment, thereby contributing to the development of chronic hyperinsulinemia, insulin resistance, dyslipidemia, and oxidative stress [22]. These conditions may be associated with cancer progression via stimulating cell proliferation, loss of epithelial integrity, angiogenesis, cell migration, and metastasis [22].

\subsection{Breast Adipose Tissue Microenvironment}

The role of the breast adipose tissue microenvironment in breast cancer development and progression is a relatively new area of research. As adipose tissue is a major component of the breast, breast cancers originate within an adipose-rich microenvironment. However, the biology of the breast adipose depot and how it changes during obesity are incompletely understood. There is known diversity in adipose depots [40], most notably between subcutaneous WAT and visceral WAT - the latter considered more metabolically active and strongly related to obesity-related comorbidities such as type 2 diabetes and cardiovascular disease $[27,49]$. Growing scientific evidence shows obesity-related conditions such as insulin resistance, type 2 diabetes [50,51], and the metabolic syndrome [52] as well as obesity-related systemic markers (e.g., leptin [53], adiponectin [54], SHBG [55], insulin and the IGF axis [56-60] and C-reactive protein [61,62]) are associated with breast cancer. How breast adipose tissue differs from other adipose depots is not fully elucidated, but it does have unique tissue-specific functions [40]. Adipose cells in the breast continually interact with breast epithelial cells by providing structural support and regulating signals [40]. In vitro and in vivo studies show that breast adipocytes are required for initial growth, branching, and maintenance of the epithelial ducts as well as functional differentiation before pregnancy [40]. Additionally, the mammary gland epithelium and adipose tissue both undergo cyclic remodeling during pregnancy, lactation, and involution [40]. The role of adipose tissue in normal mammary gland development has been proposed as a possible explanation of the inverse association observed between obesity and premenopausal breast cancer [63]. Other explanations include disruptions to the menstrual cycle [64] and lower mammographic density $[65,66]$ in obese premenopausal women.

Interactions between adipose tissue and breast epithelium may also play a role in pathological conditions of the breast such as breast cancer. For instance, emerging evidence suggests breast adipose tissue shares similar pro-inflammatory mechanisms as other adipose tissue depots including obesity-induced macrophage infiltration [22]. The pioneering work of Dannenberg and colleagues demonstrated that macrophage infiltration, as evidenced by the presence of CLS, occurs in the adipose tissue of the mouse mammary gland and in the breast of humans [67]. These crown-like structures of the breast (CLS-B) have garnered considerable interest as a histologic marker of local WAT inflammation. Given the distinct features and functions of breast adipose tissue and the close proximity to where breast cancers originate, these localized inflammatory lesions may provide unique insight into the underlying mechanisms of the obesity-breast cancer association. The rest of this review will focus on CLS-B and summarize the current epidemiologic evidence regarding their role in breast cancer development and prognosis. 


\section{Crown-Like Structures of the Breast (CLS-B): Histologic Marker of Local Inflammation}

Similar to CLS formation in other adipose depots, CLS-B are thought to form during weight gain when adipocyte hypertrophy occurs in the breast, leading to adipocyte stress and death, macrophage recruitment, and encirclement of necrotic adipocytes by macrophages in a crown-like pattern [68]. There is currently no standardized methodology for assessing CLS-B in epidemiologic studies (as discussed later in this review). Generally, CLS-B are identified by staining breast adipose tissue for a macrophage marker (e.g., CD68) using immunohistochemistry. Adipocyte encirclement by macrophages may vary from partial to complete encirclement (Figure 1). No minimum amount of encirclement has been proposed for defining CLS-B. One study used $\geq 50 \%$ encirclement for primary analyses and made comparisons using higher cut points $(\geq 75 \%$ and $\geq 90 \%)$ with few differences in conclusions by cut point used [69]. Based on the study's definition of CLS-B, a breast pathologist then enumerates CLS-B typically by visual assessment. Patient breast adipose tissue samples are often classified according to the presence or absence of CLS-B and can be further quantified using the density of CLS-B per total area of breast WAT (e.g., CLS-B $/ \mathrm{cm}^{2}$ of WAT). Previous studies have used CLS-B density for assessing the severity of WAT inflammation in the breast [69-73]. These studies used the median density value among those with CLS-B to categorize the severity of WAT inflammation with those below the median considered as having mild WAT inflammation and those above the median as having severe WAT inflammation [69-73]. Thus, cut points differ by study and no empirically-based threshold has been determined for defining clinically relevant severe WAT inflammation.

A

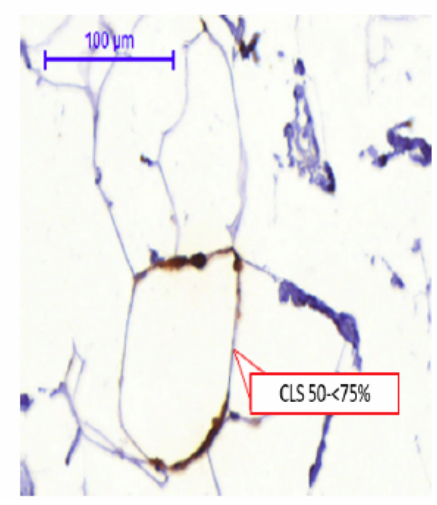

B

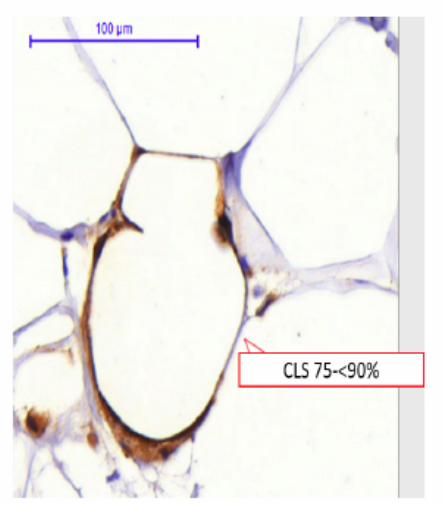

C

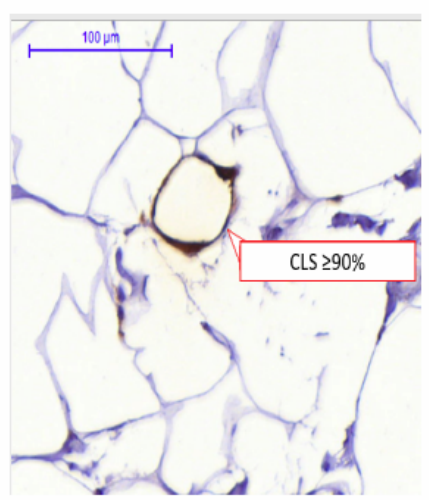

Figure 1. Whole slide digital images of anti-CD68-immunostained breast tissue captured with 3DHISTECH Panoramic Scanner 150 and analyzed using Panoramic Viewer 1.15.4 (3DHISTECH Ltd., Budapest, Hungary) from a case with CLS-B (A) CD68-immunostained tissue showing 50-<75\% adipocyte encirclement; (B) CD68-immunostained tissue showing $75-<90 \%$ adipocyte encirclement; (C) CD68-immunostained tissue showing $\geq 90 \%$ adipocyte encirclement. Adapted from Maliniak et al. 2020 [69].

Initial studies of CLS-B suggest that they create a microenvironment in the breast that is rich in pro-inflammatory cytokines and increased aromatization (Figure 2) [67,74]. This occurs through macrophage exposure to saturated fatty acids from lipolysis, which can activate Toll-like receptor 4 (TLR4) at the macrophage cell surface, leading to activation of the transcription factor nuclear factor kappa B (NF- $\mathrm{kB})$ [75]. A small pilot study $(\mathrm{n}=30)$ found that CLS-B presence was associated with elevated NF- $\mathrm{KB}$ binding activity in the breast tissue of women who received mastectomy to prevent or treat breast cancer [67]. $\mathrm{NF}-\mathrm{KB}$ regulates the expression of more than 500 genes involved in inflammation, cellular transformation, survival, proliferation, angiogenesis, metastasis, and chemo- and radiotherapy resistance [76]. Some of these include TNF $\alpha$, IL-1 $\beta$, IL-6, and COX-2-derived PGE 2 , which are related to increased aromatase activity in adipose tissue as well as other protumorigenic mechanisms $[67,73,77-79]$. The same pilot study and two other studies found 
that CLS-B were positively associated with aromatase activity in breast tissue $[67,71,72]$. One of these studies was limited to ideal-weight women (BMI $<25 \mathrm{~kg} / \mathrm{m}^{2}$ ), suggesting that local inflammation and increased aromatization (as indicated by CLS-B presence) occurs even in the absence of obesity. Another study conducted among 83 postmenopausal breast cancer patients found that CLS-B were associated with increased ratios of estrogens to androgens in breast fat and serum, indicative of increased aromatization with local and systemic effects [80].

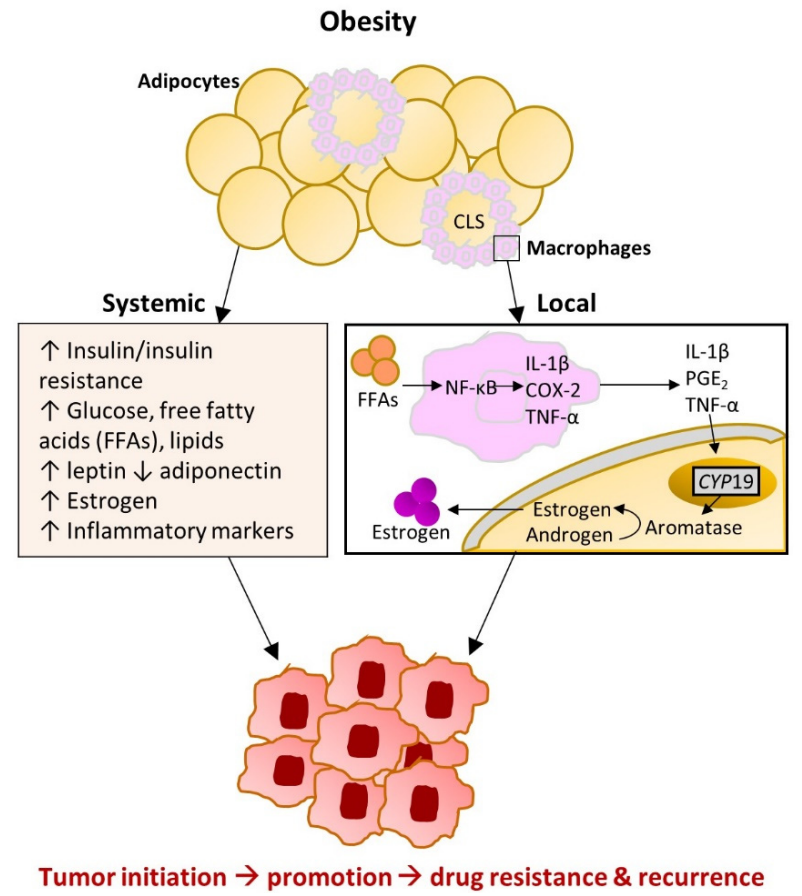

Figure 2. Hormonal and inflammatory effects of obesity-induced adipose dysfunction occurring at both the systemic and local levels. Abbreviations: CLS: crown-like structure; COX-2: cyclooxygenase2; FFAs: free fatty acids; IL: interleukin; NF-kB: nuclear factor kappa B; PGE2: prostaglandin E2; TNF- $\alpha$ : tumor necrosis factor alpha. Adapted with permission of Annual Reviews, Inc., from Iyengar NM, Hudis CA, Dannenberg AJ. Obesity and cancer: local and systemic mechanisms. Annu Rev Med. 2015; 66: 297-309; permission conveyed through Copyright Clearance Center, Inc. [74].

Furthermore, CLS-B have been associated with an unfavorable metabolic and inflammatory profile. Four studies among mostly breast cancer patients demonstrated associations between CLS-B and circulating metabolic and inflammatory markers [71,81-83]. In an analysis of 100 women who underwent mastectomy and donated fasting blood specimens at the time of surgery, CLS-B presence was associated with elevated levels of glucose, insulin, leptin, triglycerides, high-sensitivity C-reactive protein (CRP), and IL-6; CLS-B presence was associated with lower levels of HDL cholesterol and adiponectin [82]. Similar findings were observed in the three other studies $[71,81,83]$. None of these studies adjusted for BMI in their analyses; however, one study was limited to ideal-weight women, which provides some evidence that the observed associations were not entirely driven by elevated BMI among women with CLS-B [71].

Further description of the molecular pathways between CLS-B and sustained local WAT inflammation can be found in several reviews $[7,23,36,75,78]$.

\section{Potential Etiologic Drivers of CLS-B}

Epidemiologic evidence examining the role of CLS-B in breast cancer is sparse. Table 1 provides a summary of previous epidemiologic studies $(n=14)$. The majority of these were cross-sectional analyses aimed at identifying factors related to CLS-B, as reviewed below. 
Table 1. Overview of the epidemiologic studies examining CLS-B and female breast cancer.

\begin{tabular}{|c|c|c|c|c|c|c|}
\hline $\begin{array}{l}\text { First Author } \\
\text { (Year) }\end{array}$ & Study Design & $\begin{array}{l}\text { Institutions/ } \\
\text { Affiliations }\end{array}$ & $\begin{array}{l}\text { Country, } \\
\text { Race/ } \\
\text { Ethnicity } \\
\text { Distribution }\end{array}$ & Study Population & Study Years & CLS-B Analyses Conducted \\
\hline Shaik (2020) [84] & $\begin{array}{l}\text { Nested } \\
\text { case-control + } \\
\text { cross-sectional } \\
\text { analysis }\end{array}$ & $\begin{array}{l}\text { Detroit BBD } \\
\text { cohort and KTB }\end{array}$ & $\begin{array}{l}\text { USA } \\
100 \% \text { AA }\end{array}$ & $\begin{array}{l}n=84 \mathrm{BBD} \text { cases } \\
n=47 \mathrm{BBD} \text { controls } \\
n=50 \mathrm{KTB} \text { volunteers without } \\
\text { BBD or breast cancer }\end{array}$ & $\begin{array}{l}\text { BBD diagnosis: } \\
\text { 1997-2010 } \\
\text { Follow up for breast } \\
\text { cancer through } 2016\end{array}$ & $\begin{array}{ll}\text { - } & \text { Association between CLS-B and breast } \\
\text { - } & \text { CLncer among AA women with BBD } \\
\text { - } & \text { BMI and CLS-B associations } \\
\text { - } & \text { Adipocyte diameter and CLS-B } \\
\text { - } & \text { Associations between IL (another } \\
\text { inflammatory marker) with breast cancer } \\
\text { and BBD among AA women }\end{array}$ \\
\hline Carter (2017) [85] & $\begin{array}{l}\text { Nested } \\
\text { case-control + } \\
\text { cross-sectional } \\
\text { analysis }\end{array}$ & $\begin{array}{l}\text { Mayo BBD cohort } \\
\text { and KTB }\end{array}$ & $\begin{array}{l}\text { USA } \\
\text { Unknown }\end{array}$ & $\begin{array}{l}n=86 \text { BBD cases } \\
n=86 \text { BBD controls } \\
n=86 \text { KTB volunteers without } \\
\text { clinical breast abnormalities }\end{array}$ & $\begin{array}{l}\text { BBD diagnosis: } \\
\text { 1967-2001 } \\
\text { Follow up for breast } \\
\text { cancer: Unknown }\end{array}$ & $\begin{array}{l}\text { - } \\
\text { cansociation between CLS-B and breast } \\
\text { - } \quad \text { CLS-B occurrence in normal breast tissue } \\
\text { - } \quad \text { Participant and clinical characteristics } \\
\text { associated with CLS-B } \\
\text { - } \quad \text { Stromal CD68+ macrophage infiltration } \\
\text { by BBD and breast cancer status }\end{array}$ \\
\hline \multicolumn{7}{|c|}{ Breast cancer prognosis studies $(n=4)$} \\
\hline $\begin{array}{l}\text { Maliniak } \\
\text { (2020) [69] }\end{array}$ & $\begin{array}{l}\text { Cohort }+ \\
\text { cross-sectional } \\
\text { analysis }\end{array}$ & $\begin{array}{l}\text { Emory } \\
\text { University- } \\
\text { affiliated tumor } \\
\text { registries }\end{array}$ & $\begin{array}{l}\text { USA } \\
51 \% \text { AA } \\
49 \% \text { White }\end{array}$ & $\begin{array}{ll}n= & 342 \text { breast cancer patients } \\
\bullet & \text { Age } \geq 18 \text { years old } \\
- & \text { Stage I-III, invasive } \\
\bullet & \text { Underwent mastectomy } \\
-\quad & \text { No neoadjuvant treatment }\end{array}$ & $\begin{array}{l}\text { Breast cancer diagnosis: } \\
2007-2012 \\
\text { Follow up for breast } \\
\text { cancer outcomes: } 2018\end{array}$ & $\begin{array}{l}\text { Association between CLS-B and breast } \\
\text { cancer prognosis } \\
\text { - } \\
\text { Occurrence of CLS-B by race (AA vs. } \\
\text { White) } \\
\text { Participant and tumor characteristics } \\
\text { associated with CLS-B } \\
\text { - }\end{array}$ \\
\hline
\end{tabular}


Table 1. Cont.

\begin{tabular}{|c|c|c|c|c|c|c|}
\hline $\begin{array}{l}\text { First Author } \\
\text { (Year) }\end{array}$ & Study Design & $\begin{array}{l}\text { Institutions/ } \\
\text { Affiliations }\end{array}$ & $\begin{array}{l}\text { Country, } \\
\text { Race/ } \\
\text { Ethnicity } \\
\text { Distribution }\end{array}$ & Study Population & Study Years & CLS-B Analyses Conducted \\
\hline Cha (2018) [86] & $\begin{array}{l}\text { Cohort }+ \\
\text { cross-sectional } \\
\text { analysis }\end{array}$ & Yonsei University & South Korea & $\begin{array}{l}\text { Group 1: } n=56 \text { non-breast } \\
\text { cancer patients } \\
\text { - Tissue from reduction } \\
\text { mammoplasty } \\
\text { Group 2: } n=84 \text { breast cancer } \\
\text { patients } \\
\text { - Non-tumor breast tissue } \\
\text { Group 3: } n=140 \text { breast cancer } \\
\text { patients } \\
\text { - Tumor-containing breast } \\
\text { tissue }\end{array}$ & Unknown & $\begin{array}{l}\text { - Association between CLS-B and breast } \\
\text { cancer prognosis (Group } 3 \text { only) } \\
\text { Occurrence of CLS-B by breast cancer } \\
\text { status and type of tissue among breast } \\
\text { cancer patients (non-neoplastic vs. } \\
\text { neoplastic) } \\
\text { Participant and tumor characteristics } \\
\text { associated with CLS-B (Group } 3 \text { only) } \\
\text { Associations between number of } \\
\text { infiltrating CD68+ and CD163+ in } \\
\text { adipose tissue and tumor tissue with } \\
\text { CLS-B detected by CD68 and CD163 } \\
\text { (Group } 3 \text { only) }\end{array}$ \\
\hline $\begin{array}{l}\text { Koru-Sengul } \\
\text { (2016) [87] }\end{array}$ & $\begin{array}{l}\text { Cohort }+ \\
\text { cross-sectional } \\
\text { analysis }\end{array}$ & $\begin{array}{l}\text { University of } \\
\text { Miami/Jackson } \\
\text { Memorial } \\
\text { Hospital tumor } \\
\text { registry }\end{array}$ & $\begin{array}{l}\text { USA } \\
33 \% \text { Black } \\
33 \% \text { non-Black } \\
\text { Latina } \\
33 \% \text { Caucasian }\end{array}$ & $\begin{array}{l}n=150 \text { breast cancer patients } \\
\text { - } \quad \text { Stage I-IV } \\
\text { No previous exposure to } \\
\text { chemotherapy, } \\
\text { radiotherapy, or hormonal } \\
\text { therapy }\end{array}$ & $\begin{array}{l}\text { Cases obtained: } \\
\text { 1978-1997 } \\
\text { Followed for at least } 5 \\
\text { years }\end{array}$ & 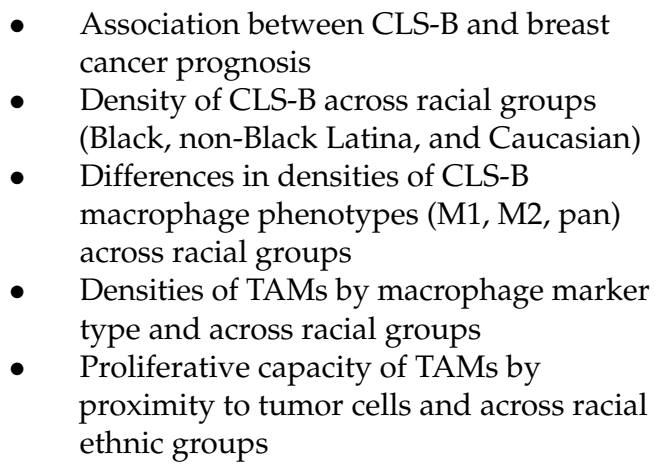 \\
\hline
\end{tabular}


Table 1. Cont.

\begin{tabular}{|c|c|c|c|c|c|c|}
\hline $\begin{array}{l}\text { First Author } \\
\text { (Year) }\end{array}$ & Study Design & $\begin{array}{l}\text { Institutions/ } \\
\text { Affiliations }\end{array}$ & $\begin{array}{l}\text { Country, } \\
\text { Race/ } \\
\text { Ethnicity } \\
\text { Distribution }\end{array}$ & Study Population & Study Years & CLS-B Analyses Conducted \\
\hline \multicolumn{7}{|c|}{ Cross-sectional studies of CLS-B $(n=8)$} \\
\hline $\begin{array}{l}\text { Greenlee } \\
\text { (2018) [70] }\end{array}$ & Cross-sectional & $\begin{array}{l}\text { Columbia } \\
\text { University } \\
\text { Medical Center }\end{array}$ & $\begin{array}{l}\text { USA } \\
100 \% \text { Hispanic }^{a}\end{array}$ & $\begin{array}{l}n=91 \text { breast cancer patients } \\
\text { - } \quad \text { Stage } 0-\text { III } \\
\text { - } \quad \text { Underwent mastectomy } \\
\text { 16\% had neoadjuvant } \\
\quad \text { chemotherapy }\end{array}$ & Mastectomy: 2007-2012 & $\begin{array}{l}\text { - Occurrence of CLS-B among } \\
\text { - Hispanic/Latina breast cancer patients } \\
\text { Participant and tumor characteristics } \\
\text { - } \quad \text { Adsociated with CLS-B } \\
\text { - } \quad \text { Liver function biomarkers and CLS-B }\end{array}$ \\
\hline $\begin{array}{l}\text { Iyengar } \\
\text { (2018) [81] }\end{array}$ & Cross-sectional & $\begin{array}{l}\text { National Taiwan } \\
\text { University } \\
\text { Hospital and } \\
\text { MSKCC }\end{array}$ & $\begin{array}{l}\text { Taiwan } \\
\text { USA } \\
\text { 100\% Caucasian }\end{array}$ & $\begin{array}{l}n=72 \text { Taiwanese breast cancer } \\
\text { patients } \\
\text { - } \quad \text { Non-metastatic } \\
\text { - } \quad \text { Underwent mastectomy } \\
\text { - } \quad n=267 \text { US Caucasian } \\
\quad \text { patients } \\
\text { - Underwent mastectomy }\end{array}$ & $\begin{array}{l}\text { Mastectomy: 2011-2016 } \\
\text { (Taiwanese); 2011-2013 } \\
\text { (US Caucasian) }\end{array}$ & $\begin{array}{l}\text { Comparisons of CLS-B and breast } \\
\text { adipocyte size in Taiwanese vs.US } \\
\text { Caucasian women } \\
\text { Participant and tumor characteristics } \\
\text { associated with CLS-B (Taiwanese only) } \\
\text { Body composition (body fat, VAT, SAT) } \\
\text { factors associated with CLS-B (Taiwanese } \\
\text { only) } \\
\text { Circulating metabolic and inflammatory } \\
\text { markers associated with CLS-B } \\
\text { (Taiwanese only) } \\
\text { Adipocyte diameter and CLS-B }\end{array}$ \\
\hline
\end{tabular}


Table 1. Cont.

\begin{tabular}{|c|c|c|c|c|}
\hline $\begin{array}{l}\text { First Author } \\
\text { (Year) }\end{array}$ & Study Design & $\begin{array}{l}\text { Institutions/ } \\
\text { Affiliations }\end{array}$ & $\begin{array}{l}\text { Country, } \\
\text { Race/ } \\
\text { Ethnicity } \\
\text { Distribution }\end{array}$ & Study Population \\
\hline $\begin{array}{l}\text { Iyengar } \\
\text { (2017) [71] }\end{array}$ & Cross-sectional & MSKCC & $\begin{array}{l}\text { USA } \\
76 \% \text { Caucasian } \\
9 \% \text { Black, Asian, } \\
\text { or Other } \\
14 \% \text { Unknown }\end{array}$ & $\begin{array}{l}n=72 \text { patients (mostly breast } \\
\text { cancer) } \\
\text { - Normal weight } \\
\quad\left(\text { BMI }<25 \mathrm{~kg} / \mathrm{m}^{2}\right) \\
\text { - Underwent mastectomy }\end{array}$ \\
\hline
\end{tabular}

Study Years

\section{Mastectomy: 2011-2013}

- Occurrence of CLS-B in normal weight women

- Participant and tumor characteristics associated with CLS-B

- Circulating metabolic and inflammatory markers associated with CLS-B

- Circulating leptin and CLS-B, aromatase expression, and adipocyte diameter associations

- $\quad$ Adipocyte diameter and CLS-B

- Aromatase activity and CLS-B, BMI, and adipocyte diameter associations

$n=83$ breast cancer patients

- Invasive breast cancer

- Postmenopausal and not taking hormone replacement

Study recruitment:

2000-2003

- Underwent surgery

- No neoadjuvant treatment
- Participant and tumor characteristics associated with CLS-B and number of CD68-positive cells

- CLS-B associations with concentrations and ratios of sex-steroid hormones in breast adipose tissue vs. systemic circulation

- $\quad$ BMI, WHR, \% truncal fat and CLS-B associations overall and by menopausal status

$=107$ breast cancer patient

Energy Balance

Vaysse (2017) [83] Cross-sectional and Breast Norway
- $\quad$ Aged 25-75 years

- Stage I-II, invasive

- $71 \%$ underwent breast conserving surgery
Unknown

Circulating metabolic and inflammatory markers associated with CLS-B overall and by menopausal status

Adipocyte diameter and CLS-B 
Table 1. Cont.

\begin{tabular}{|c|c|c|c|c|c|c|}
\hline $\begin{array}{l}\text { First Author } \\
\text { (Year) }\end{array}$ & Study Design & $\begin{array}{l}\text { Institutions/ } \\
\text { Affiliations }\end{array}$ & $\begin{array}{l}\text { Country, } \\
\text { Race/ } \\
\text { Ethnicity } \\
\text { Distribution }\end{array}$ & Study Population & Study Years & CLS-B Analyses Conducted \\
\hline Brown (2017) [72] & Cross-sectional & MSKCC & USA & $\begin{array}{l}n=161 \text { patients (mostly breast } \\
\text { cancer) } \\
\text { - } \quad \text { Aged } 27-74 \text { years } \\
\text { - } \quad \text { Underwent mastectomy }\end{array}$ & Unknown & $\begin{array}{l}\text { - } \quad \text { Menopause and CLS-B association } \\
\text { - The effect of menopause on CLS-B and } \\
\text { aromatase expression associations }\end{array}$ \\
\hline $\begin{array}{l}\text { Iyengar } \\
\text { (2015) [73] }\end{array}$ & Cross-sectional & MSKCC & $\begin{array}{l}\text { USA } \\
86 \% \text { White } \\
7 \% \text { Black } \\
6 \% \text { Asian }\end{array}$ & $\begin{array}{l}n=237 \text { patients (mostly breast } \\
\text { cancer) } \\
\text { - } \quad \text { Aged 22-90 years } \\
\text { - } \quad \text { Underwent mastectomy } \\
\text { - } \quad 14 \% \text { received preoperative } \\
\text { chemotherapy } \\
\text { - } 39 \% \text { of those tested had } \\
\quad \text { BRCA1/2 mutation }\end{array}$ & Mastectomy: 2011-2013 & $\begin{array}{l}\text { - } \quad \text { Participant and tumor characteristics } \\
\text { associated with CLS-B } \\
\text { - } \quad \text { Adipocyte diameter and CLS-B } \\
\text { - Comparison of CLS-B status between } \\
\text { bilateral breasts } \\
\text { - Comparison of CLS-B status with } \\
\text { abdominal CLS status }\end{array}$ \\
\hline Morris (2011) [67] & $\begin{array}{l}\text { Cross-sectional } \\
\text { (pilot study) }\end{array}$ & MSKCC & USA & $\begin{array}{l}n=30 \text { patients (mostly breast } \\
\text { cancer) } \\
\bullet \quad \text { Aged } 26-70 \text { years } \\
\bullet \quad \text { Underwent mastectomy }\end{array}$ & Enrolled: 2010 & $\begin{array}{ll}\text { - } & \text { BMI and CLS-B association } \\
\text { - } & \text { Adipocyte diameter and CLS-B } \\
\text { - } & \text { Aromatase expression and activity and } \\
& \text { CLS-B } \\
\text { - } & \text { NF-kB binding activity and CLS-B }\end{array}$ \\
\hline
\end{tabular}

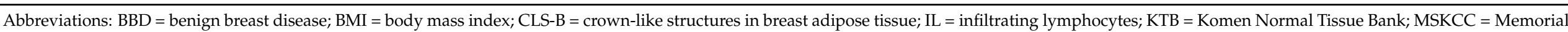

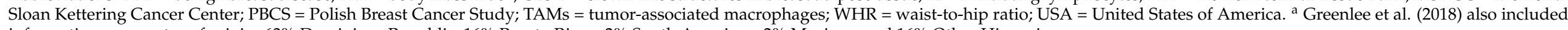
information on country of origin: 63\% Dominican Republic, 16\% Puerto Rican, 2\% South American, 2\% Mexican, and 16\% Other Hispanic 


\subsection{Obesity and CLS-B}

Cross-sectional studies have consistently demonstrated a strong, positive association between adiposity, typically measured using BMI, and CLS-B presence and density in numerous independent populations of predominantly breast cancer patients (Table 2) $[73,80,81,83,87]$. For example, one study including 237 patients who underwent mastectomy for breast cancer prevention or treatment, detected CLS-B in $90 \%$ of obese patients (BMI $\geq 30 \mathrm{~kg} / \mathrm{m}^{2}$ ), $53 \%$ of overweight patients (BMI $25-<30 \mathrm{~kg} / \mathrm{m}^{2}$ ), and $34 \%$ of ideal-weight patients $\left(\mathrm{BMI}<25 \mathrm{~kg} / \mathrm{m}^{2}\right)$ [73]. The relatively high proportion of ideal-weight women with CLS-B suggests that even among lean women, adipocyte hypertrophy may be relevant for breast carcinogenesis [71].

Few studies have examined the association between CLS-B and other measures of adiposity. A Norwegian study including 107 patients with early-stage breast cancer examined associations between CLS-B and three adiposity measures (BMI, WHR, and percent truncal fat) [83]. It found that women with a BMI $\geq 30 \mathrm{~kg} / \mathrm{m}^{2}$ were almost seven times as likely to have CLS-B present as women with a BMI $<25 \mathrm{~kg} / \mathrm{m}^{2}$, although the association was imprecise as demonstrated by the wide $95 \%$ confidence interval (OR: 6.9; 95\%CI: 1.35 to 35.0) [83]. Strong associations were also found for WHR ( $>0.85 \mathrm{vs.} \leq 0.85 ;$ OR: $3.26,95 \% \mathrm{CI}$ : 1.35 to 7.85 ) and percent truncal fat (per standard deviation; OR: $3.58,95 \% \mathrm{CI}$ : 2.00 to 6.44 ). These associations were generally consistent across menopausal status except for percent truncal fat, which showed a stronger association among premenopausal women [83]. Another study with body composition measurements among Taiwanese breast cancer patients found that women with CLS-B had a higher body fat percentage (33\% vs. $27.5 \%$; $p<0.01$ ), greater visceral ( $2.7 \mathrm{~kg}$ vs. $1.5 \mathrm{~kg} ; p<0.01)$, and subcutaneous adipose tissue (20.1 kg vs. $14 \mathrm{~kg} ; p<0.01$ ) compared with patients without CLS-B [81].

At the tissue level, several studies have demonstrated that CLS-B are positively correlated with breast adipocyte diameter, as would be expected given their association with obesity $[67,69-71,73,81,83,84]$. Even among ideal-weight women, a positive, linear association was found between CLS-B density and adipocyte diameter $(\rho=0.36 ; p<0.01)$ [71].

There is little evidence relating obesity with CLS-B in study populations of women without breast cancer due to the difficulty in obtaining breast adipose tissue for such analyses. In one case-control study including patients with benign breast disease (BBD) that was nested within the Mayo BBD cohort and also included a group of women that donated normal breast tissue to the Komen Normal Tissue Bank (KTB), CLS-B were detected in 7\% of women with BMI $<25 \mathrm{~kg} / \mathrm{m}^{2}, 13 \%$ with BMI 25-29, and $29 \%$ with BMI $\geq 30$ ( $\left.p=0.0005\right)$ [85]. This association was primarily driven by the women with BBD-as only $3 \%$ of the KTB donors were found to have CLS-B present in their tissue samples [85]. In another case-control study including African American women from the Detroit BBD Cohort and African American KTB donors, the authors reported no association between BMI and CLS-B [84]. 
Table 2. Cross-sectional analyses examining the association between obesity and CLS-B.

\begin{tabular}{|c|c|c|c|c|c|c|}
\hline First Author (Year) & Patient Study Population & \multicolumn{2}{|c|}{$\%$ CLS-B+ by BMI $\left(\mathrm{kg} / \mathrm{m}^{2}\right)$ Group } & $\begin{array}{l}\text { Association between BMI and CLS-B } \\
\text { aOR }(95 \% \mathrm{CI}) \text { if Presented }\end{array}$ & $\begin{array}{l}\text { Association with Other } \\
\text { Adiposity Measures }\end{array}$ & $\begin{array}{l}\text { Direction of } \\
\text { Association: } \\
\text {-/Null/t+ }\end{array}$ \\
\hline Shaik (2020) [84] & BBD + Komen Normal Tissue Bank & NR & & Not associated $(p>0.1)$ & & Null \\
\hline Carter (2017) [85] & BBD + Komen Normal Tissue Bank & $\begin{array}{l}\text { BMI <25: } \\
\text { BMI 25-<30: } \\
\text { BMI } \geq 30 \text { : }\end{array}$ & $\begin{array}{l}7 \% \\
13 \% \\
29 \%\end{array}$ & & & + \\
\hline Maliniak (2020) [69] & Breast cancer & $\begin{array}{l}\text { BMI }<25 \text { : } \\
\text { BMI 25-<30: } \\
\text { BMI } \geq 30 \text { : }\end{array}$ & $\begin{array}{l}16 \% \\
29 \% \\
45 \%\end{array}$ & $\begin{array}{l}\text { Reference } \\
2.34(1.17 \text { to } 4.70) \\
4.73(2.48 \text { to } 9.01)\end{array}$ & & + \\
\hline Cha (2018) ${ }^{a}[86]$ & Breast cancer & $\begin{array}{l}\mathrm{BMI}<25: \\
\mathrm{BMI} \geq 25\end{array}$ & $\begin{array}{l}15 \% \\
27 \%\end{array}$ & & & + \\
\hline Greenlee (2018) [70] & Breast cancer & $\begin{array}{l}\text { BMI 18.5-<25: } \\
\text { BMI 25-<30: } \\
\text { BMI 30-<35: } \\
\text { BMI } \geq 35 \text { : }\end{array}$ & $\begin{array}{l}24 \% \\
34 \% \\
57 \% \\
65 \%\end{array}$ & & & + \\
\hline Iyengar $(2018)^{b}[81]$ & Breast cancer & $\begin{array}{l}\text { BMI <23: } \\
\text { BMI 23-<27.5: } \\
\text { BMI } \geq 27.5:\end{array}$ & $\begin{array}{l}24 \% \\
48 \% \\
76 \%\end{array}$ & & $\begin{array}{l}\text { Body fat, VAT, and SAT (all } \\
p<0.01 \text { ) }\end{array}$ & + \\
\hline Iyengar (2017) [71] & Mostly breast cancer & All BMI < 25: & $39 \%$ & $\begin{array}{l}\text { CLS-B- vs. CLS-B + } \\
\text { median BMI: } 21.8 \text { vs. } 23.0, p=0.04\end{array}$ & & + \\
\hline Mullooly (2017) [80] & Breast cancer & $\begin{array}{l}\text { BMI <25: } \\
\text { BMI 25-<30: } \\
\text { BMI } \geq 30 \text { : }\end{array}$ & $\begin{array}{l}17 \% \\
36 \% \\
54 \%\end{array}$ & $\begin{array}{l}\text { Reference } \\
1.93(0.50 \text { to } 7.40) \\
4.63(1.08 \text { to } 19.83)\end{array}$ & & + \\
\hline Vaysse (2017) [83] & Breast cancer & $\begin{array}{l}\text { BMI <25: } \\
\text { BMI 25-<30: } \\
\text { BMI } \geq 30 \text { : }\end{array}$ & NR & $\begin{array}{l}\text { Reference } \\
3.2(1.28 \text { to } 8.15) \\
6.9(1.35 \text { to } 35.0)\end{array}$ & $\begin{array}{l}\text { WHR and \% truncal fat (all } \\
p<0.05 \text { ) }\end{array}$ & + \\
\hline Iyengar $(2016)^{c}[82]$ & Breast cancer & $\begin{array}{l}\text { BMI <25: } \\
\text { BMI 25-<30: } \\
\text { BMI } \geq 30 \text { : }\end{array}$ & $\begin{array}{l}23 \% \\
33 \% \\
67 \%\end{array}$ & & & + \\
\hline Iyengar (2015) [73] & Mostly breast cancer & $\begin{array}{l}\text { BMI }<25 \\
\text { BMI 25-<30: } \\
\text { BMI } \geq 30 \text { : }\end{array}$ & $\begin{array}{l}34 \% \\
53 \% \\
90 \%\end{array}$ & & $\begin{array}{l}\text { CLS concordance between } \\
\text { breast and abdominal SAT } \\
(p=0.12)\end{array}$ & + \\
\hline
\end{tabular}

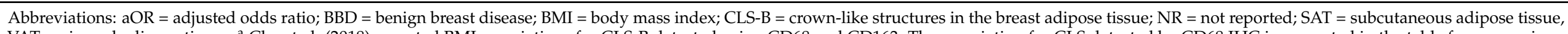

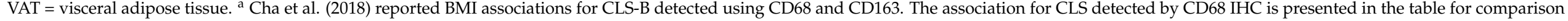

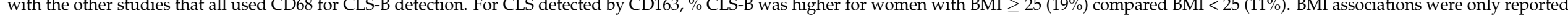

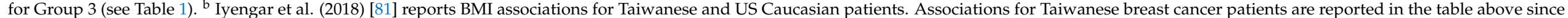

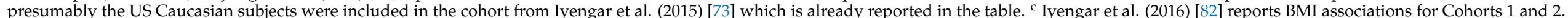

Associations for Cohort 2 are reported in the table above since the subjects in Cohort 1 are a sample of patients from Iyengar et al. (2015) [73] which is already reported in the table. 


\subsection{Other Factors and CLS-B}

Few clinical and lifestyle factors beyond BMI have been identified as associated with CLS-B (Table 3). In several investigations, older age and postmenopausal status have been linked to higher prevalence of CLS-B detection $[69,70,73,80,86]$. This relation could be due to increased breast fat percentage [88] and/or alterations to adipose tissue [89] that occur with age and menopausal transition. One study compared 102 premenopausal and 59 postmenopausal women who underwent mastectomy for breast cancer treatment or prevention and found that postmenopausal women were more likely to have a higher BMI, larger breast adipocytes, severe breast WAT inflammation as measured by the number of CLS-B $/ \mathrm{cm}^{2}$, and greater local aromatase activity than premenopausal women [72].

Table 3. Summary of the evidence between CLS-B and select patient and tumor characteristics.

\begin{tabular}{|c|c|c|}
\hline First Author (Year) & N Studies & Summary of Evidence \\
\hline \multicolumn{3}{|l|}{ Patient characteristics } \\
\hline Obesity & 11 studies $[69-71,73,80-86]$ & $\begin{array}{l}\text { Strong positive association in studies of breast cancer } \\
\text { patients (see Table 2); inconclusive evidence for BBD } \\
\text { patients and women without BBD or breast cancer }\end{array}$ \\
\hline Age & 8 studies $[69-71,73,80,81,85,86]$ & $\begin{array}{l}\text { Positive trend with age in studies of breast cancer patients } \\
\text { although majority of associations were not statistically } \\
\text { significant; no association observed between age and CLS-B } \\
\text { among BBD patients [85] }\end{array}$ \\
\hline Menopausal status & 6 studies $[69-73,81]$ & $\begin{array}{l}\text { Positive trend with postmenopausal status among breast } \\
\text { cancer patients although majority of associations were not } \\
\text { statistically significant }\end{array}$ \\
\hline Race/ethnicity & 4 studies $[69,70,81,87]$ & $\begin{array}{l}\text { Evidence of greater CLS-B density among Black breast } \\
\text { cancer patients in } n=2 \text { studies }[69,83] \text { (no association when } \\
\text { adjusting for BMI in the one study [69] with this } \\
\text { information); No strong evidence of differences in CLS-B } \\
\text { detection by country of origin among Hispanic/Latina } \\
\text { patients [70] or when comparing Taiwanese to US } \\
\text { Caucasian patients [80] }\end{array}$ \\
\hline Smoking status & 2 studies $[69,70]$ & $\begin{array}{l}\text { Positive trend with current smoking status in breast cancer } \\
\text { patients but inconclusive (very few current smokers in } \\
\text { both studies) }\end{array}$ \\
\hline Age at menopause & 2 studies $[69,80]$ & Inconclusive evidence \\
\hline Reproductive factors & 2 studies $[69,80]$ & Inconclusive evidence \\
\hline Family history of breast cancer & 2 studies $[69,80]$ & Inconclusive evidence \\
\hline \multicolumn{3}{|l|}{ Tumor characteristics } \\
\hline Molecular subtype & 6 studies $[69-71,73,80,86]$ & $\begin{array}{l}\text { No/little evidence for differences by ER status, PR status, or } \\
\text { other tumor subtypes observed }\end{array}$ \\
\hline Nodal status & 4 studies $[69,80,82,86]$ & $\begin{array}{l}\text { Some evidence suggesting association with lymph } \\
\text { node-negative disease but all together inconclusive }\end{array}$ \\
\hline Grade & 4 studies $[69,80,82,86]$ & Inconclusive evidence \\
\hline Stage & 3 studies $[69,70,86]$ & Inconclusive evidence \\
\hline
\end{tabular}

Abbreviations: $\mathrm{BBD}=$ benign breast disease; CLS-B = crown-like structures in the breast adipose tissue; ER = estrogen receptor; $\mathrm{PR}=$ progesterone receptor.

Race/ethnicity has also been explored as a possible driver of CLS-B due to the known differences in the prevalence of obesity, obesity-related comorbidities, and visceral fat accumulation by race/ethnicity [90]. Evidence of greater CLS-B density among Black breast cancer patients was observed in two studies [69,87]; however, the association attenuated after BMI adjustment in one investigation [69]. The other study did not obtain patient- 
level BMI data. Other research shows no evidence of differences in CLS-B detection by country of origin among Hispanic/Latina patients [70], or when comparing Taiwanese to US Caucasian patients [81]. Notably, the positive associations between BMI and CLS-B were consistent across these various racial/ethnic groups [69,81].

Associations with other patient factors (e.g., smoking status, reproductive factors, family history of breast cancer) and tumor characteristics are largely inconclusive due to the scarcity of evidence for these other factors.

\section{CLS-B and Incident Breast Cancer}

There have been two studies examining the association between CLS-B and breast cancer incidence (Table 4), both case-control studies nested within BBD cohorts [84,85]. The first, conducted in the Mayo BBD cohort-a large cohort of women with biopsy-proven BBD—included 86 cases (BBD patients with breast cancer) and 86 age-matched controls (BBD patients without breast cancer) [85]. CLS-B were assessed in the index BBD biopsy (before breast cancer diagnosis for cases) and found to be more frequent among cases $(24 \%)$ than controls $(19 \%)$. The association was most robust when examining $>5$ CLSB/sample (adjusted OR: 6.8, 95\%CI: 1.4 to 32.4) [85]. The second investigation included 55 cases and 47 controls nested within the Detroit BBD cohort [84]. All women were African American. This study similarly found more frequent CLS-B among cases (67\%) compared with controls (40\%), and a strong association for $\geq 5$ CLS-B/sample (adjusted OR: 4.99 , 95\%CI: 1.32 to 18.9) [84]. There are several limitations to these studies as both had small sample sizes, resulting in a large amount of uncertainty in the effect estimates. In both studies, models adjusted for a small number of potential confounders (e.g., adipose area, histologic impression, and BMI in one study [85]). Finally, since these studies were both conducted among women with BBD, it is unknown whether the results can be generalized to women without BBD. 
Table 4. Epidemiologic evidence examining CLS-B as a potential driver of female breast cancer incidence and prognosis.

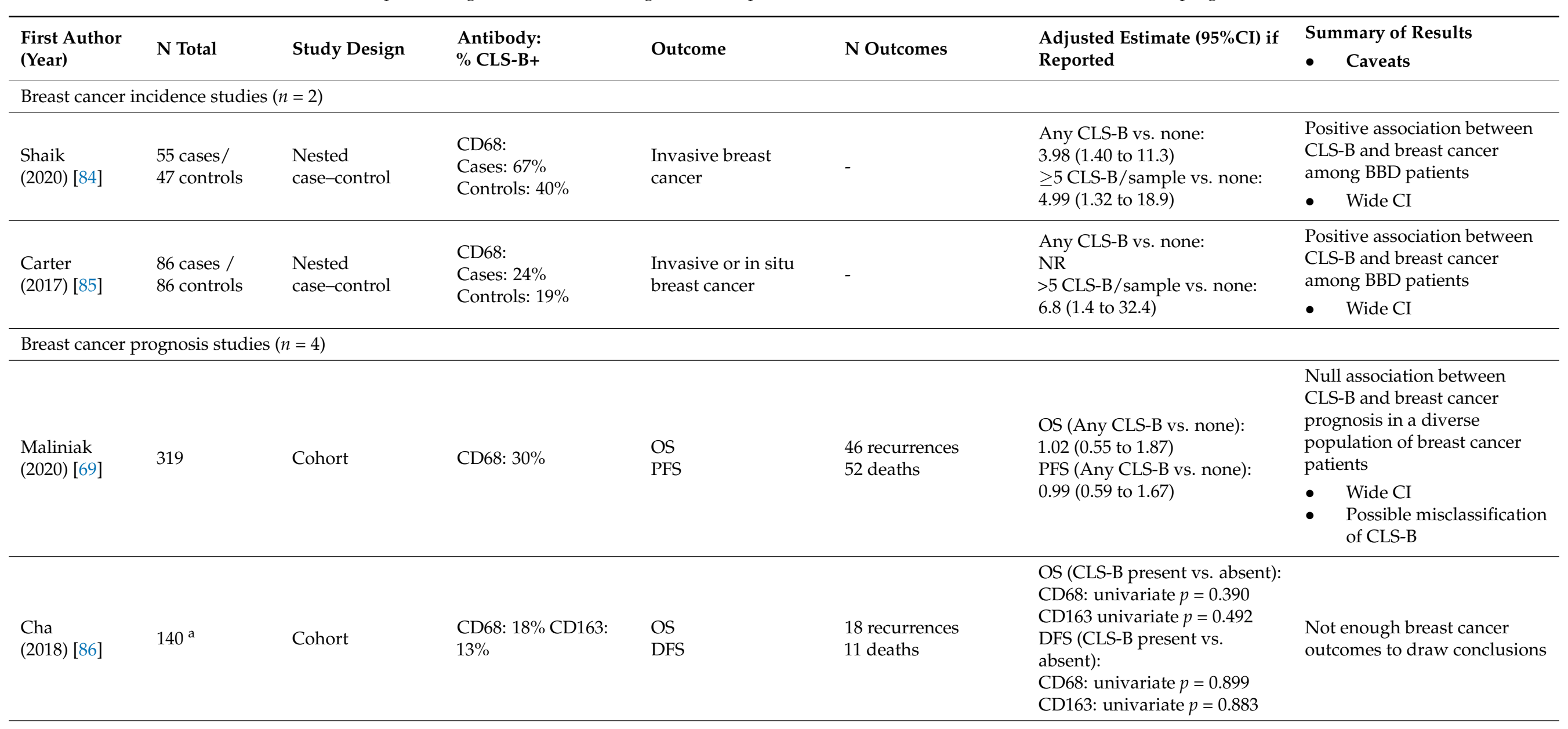


Table 4. Cont.

\begin{tabular}{|c|c|c|c|c|c|c|c|}
\hline $\begin{array}{l}\text { First Author } \\
\text { (Year) }\end{array}$ & N Total & Study Design & $\begin{array}{l}\text { Antibody: } \\
\text { \% CLS-B+ }\end{array}$ & Outcome & N Outcomes & $\begin{array}{l}\text { Adjusted Estimate }(95 \% \mathrm{CI}) \text { if } \\
\text { Reported }\end{array}$ & $\begin{array}{l}\text { Summary of Results } \\
\text { - Caveats }\end{array}$ \\
\hline $\begin{array}{l}\text { Koru-Sengul } \\
\text { (2016) [87] }\end{array}$ & 150 & Cohort & $\begin{array}{l}\text { CD163: NR CD40: } \\
\text { NR CD206: NR }\end{array}$ & $\begin{array}{l}\text { OS } \\
\text { PFS }\end{array}$ & $\begin{array}{l}83 \text { recurrences } \\
88 \text { deaths }\end{array}$ & $\begin{array}{l}\text { OS (density of CLS): } \\
\text { CD163: } 2.14 \text { (0.46 to } 9.96)^{b} \\
\text { CD40: } 9.14(1.00 \text { to } 83.60)^{b} \\
\text { CD206: } 0.65 \text { (0.03 to } 12.58)^{b} \\
\text { PFS (density of CLS): } \\
\text { CD163: } 2.30 \text { (0.66 to } 8.03)^{b} \\
\text { CD40: } 4.12(0.49 \text { to } 34.92)^{b} \\
\text { CD206: } 1.16(0.09 \text { to } 14.28)^{b}\end{array}$ & $\begin{array}{l}\text { Positive association between } \\
\text { CLS-B and breast cancer } \\
\text { prognosis } \\
\text { - Wide CI } \\
\text { - } \quad \text { Varied by antibody used } \\
\text { for detecting CLS-B } \\
\text { - Assessed CLS-B in tumor } \\
\text { tissue }\end{array}$ \\
\hline $\begin{array}{l}\text { Iyengar } \\
(2016) \text { [82] }\end{array}$ & 127 & $\begin{array}{l}\text { Case-only } \\
\text { analysis }\end{array}$ & CD68: $41 \%$ & $\begin{array}{l}\text { Average time to } \\
\text { distant recurrence }\end{array}$ & $\begin{array}{l}127 \text { recurrences } \\
99 \text { deaths }\end{array}$ & $\begin{array}{l}\text { Any CLS-B vs. none: } \\
1.83 \text { (1.07 to } 3.13)\end{array}$ & $\begin{array}{l}\text { Positive association between } \\
\text { CLS-B and breast cancer } \\
\text { prognosis } \\
\text { - Select population of } \\
\text { patients that all } \\
\text { developed metastatic } \\
\text { disease }\end{array}$ \\
\hline
\end{tabular}

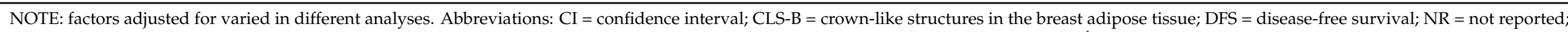

OS = overall survival; PFS = progression-free survival. ${ }^{a}$ Cha et al. (2018) [86] only reported OS and DFS associations for Group 3 (see Table 1). ${ }^{b} 90 \%$ CIs reported 


\section{CLS-B as a Potential Driver of Prognosis}

Four studies included follow-up data to examine the impact of CLS-B detection at diagnosis on clinical outcomes (Table 4) $[69,82,86,87]$. Results have been inconsistent and conclusions limited due to lack of power/precision, varying study methods, and differences in study populations. Iyengar et al. conducted the first study examining CLS-B and prognosis in a study population of patients that all developed distant recurrence. CD68 (a pan-macrophage marker) was used to identify CLS-B in non-tumor-containing breast adipose tissue. In this cohort, CLS-B presence was associated with shortened average time to distant recurrence (adjusted HR: 1.83, 95\%CI: 1.07 to 3.13) [82]. However, because this was a case-only analysis in which selection was based on having developed the outcome of interest, interpretability and generalizability of results are limited [91]. The second study by Koru-Sengul et al. assessed CLS-B using three macrophage markers for detection in the fat portions of tumor-containing tissue (CD206 = M2 marker, CD40 = M1 marker, and CD163 = pan-macrophage marker) [87]. They observed positive associations with worse overall survival for density of CLS detected by CD40 (adjusted HR: 9.14, 90\%CI: 1.00 to 83.60) and CD163 (adjusted HR: 2.14, 95\%CI: 0.46 to 9.96) but not CD206 (adjusted HR: 0.65, $90 \%$ CI: 0.03 to 12.58 ) although all associations were very imprecise [87]. Associations for progression-free survival were similarly imprecise but positive regardless of macrophage marker (e.g., for CD40: adjusted HR: 4.21, 90\%CI: 0.49 to 34.92) [87]. A study by Cha et al. used CD68 and CD163 (described as an M2 macrophage marker in this study) to detect CLS-B in three groups of patients with breast adipose tissue (Group 1 from reduction mammoplasty; Group 2 from non-neoplastic tissue from resected breast cancer specimens; Group 3 from breast cancer tissue specimens) [86]. However, this study had few recurrences $(n=18)$ and deaths $(n=11)$ among breast cancer patients and did not report effect estimates for associations between CLS-B and clinical outcomes [86]. In the largest study to date, we recently investigated this topic in a diverse cohort of Black and White breast cancer patients unrestricted to outcome and used CD68 to identify CLS-B in non-tumor-containing breast adipose tissue [69]. We found no evidence of an association between CLS-B with overall (adjusted HR: 1.02, 95\%CI: 0.55 to 1.87) or progression-free survival (adjusted HR: $0.99,95 \%$ CI: 0.59 to 1.67$)$. Despite this being the most methodologically robust study to date, estimates remained imprecise and may have been biased toward the null due to misclassification of CLS-B; only one tissue specimen per patient was used for assessing CLS-B as compared to five tissue specimens per patient in the study by lyengar et al. [69,82]. Given the limitations and scarcity of evidence, it is still unclear whether CLS-B could serve as a prognostic marker in breast cancer.

\section{Future Directions}

The role of CLS-B in breast cancer is not established. We have identified three broad areas for future work in this area: (1) establishing standards for CLS-B assessment; (2) assessing the role of CLS-B in breast cancer incidence, and (3) assessing the role of CLS-B on therapeutic effectiveness and breast cancer prognosis.

\subsection{Methodology in CLS-B Assessment}

Standards for CLS-B assessment based on empirical evidence are needed for a broader assessment in epidemiologic studies and to facilitate comparisons across studies. Different methods have been used across epidemiologic studies to date (Table 5). Questions need to be answered regarding the number of tissue specimens required to adequately assess CLS-B; the most clinically relevant macrophage marker(s) for identifying CLS-B; and whether CLS-B can be appropriately evaluated in tissue samples beyond those obtained from mastectomy. 
Table 5. Summary of CLS-B assessment methods by epidemiologic study.

\begin{tabular}{|c|c|c|c|c|}
\hline First Author (Year) & Tissue Specimen & $\begin{array}{l}\text { Tissue } \\
\text { Specimens } \\
\text { per Subject }\end{array}$ & Antibody & $\%$ CLS-B+ \\
\hline \multicolumn{5}{|c|}{ Breast cancer incidence studies $(n=2)$} \\
\hline Shaik (2020) [84] & $\begin{array}{l}\text { BBD: FFPE BBD biopsy tissue } \\
\text { KTB donors: FFPE percutaneous } \\
\text { needle biopsy tissue }\end{array}$ & 1 & CD68 & $\begin{array}{l}\text { BBD Cases: } 67 \% \\
\text { BBD Controls: } 40 \% \\
\text { KTB donors: } 18 \%\end{array}$ \\
\hline Carter (2017) [85] & $\begin{array}{l}\text { BBD: FFPE BBD biopsy tissue } \\
\text { KTB donors: FFPE normal breast tissue }\end{array}$ & 1 & CD68 & $\begin{array}{l}\text { BBD Cases: } 24 \% \\
\text { BBD Controls: } 19 \% \\
\text { KTB donors: } 3 \%\end{array}$ \\
\hline \multicolumn{5}{|c|}{ Breast cancer prognosis studies $(n=4)$} \\
\hline Maliniak (2020) [69] & FFPE non-tumor tissue & 1 & CD68 & $\begin{array}{l}\text { Overall: } 30 \% \\
\text { AA: } 32 \% \\
\text { White: } 29 \%\end{array}$ \\
\hline Cha (2018) [86] & $\begin{array}{l}\text { Group 1: FFPE reduction } \\
\text { mammoplasty } \\
\text { Group 2: FFPE non-tumor tissue } \\
\text { Group 3: FFPE tumor tissue }\end{array}$ & Unknown & $\begin{array}{l}\text { CD68 } \\
\text { CD163 }\end{array}$ & $\begin{array}{l}\text { CD68, CD } 163 \\
\text { Group 1: } 2 \%, 2 \% \\
\text { Group 2: } 0 \%, 0 \% \\
\text { Group 3: } 18 \%, 13 \%\end{array}$ \\
\hline Koru-Sengul (2016) [87] & FFPE tumor tissue & 1 & $\begin{array}{l}\text { CD163 } \\
\text { CD206 } \\
\text { CD40 }\end{array}$ & $\begin{array}{l}\text { Density of CLS: CD163, CD206, CD40 } \\
\text { Mean (SD) } \\
\text { All: } 0.06(0.14) ; 0.03(0.07) ; 0.01(0.07) \\
\text { Black: } 0.11(0.22) ; 0.04(0.09) ; 0.02(0.11) \\
\text { NBLA: } 0.05(0.08) ; 0.03(0.05) ; 0(0) \\
\text { CA: } 0.03(0.07) ; 0.02(0.06) ; 0(0.02)\end{array}$ \\
\hline Iyengar (2016) [82] & FFPE non-tumor tissue & 5 & CD68 & $41 \%$ \\
\hline \multicolumn{5}{|c|}{ Cross-sectional studies of CLS-B $(n=8)$} \\
\hline Greenlee (2018) [70] & FFPE non-tumor tissue & 5 & CD68 & $45 \%$ \\
\hline Iyengar (2018) [81] & FFPE non-tumor tissue & 5 & CD68 & $\begin{array}{l}\text { Taiwanese: } 43 \% \\
\text { US Caucasian: } 55 \%\end{array}$ \\
\hline $\begin{array}{l}\text { Iyengar (2017) [71] } \\
\text { Mullooly (2017) [80] }\end{array}$ & $\begin{array}{l}\text { FFPE non-tumor tissue } \\
\text { FFPE non-tumor tissue }\end{array}$ & $\begin{array}{l}5 \\
1\end{array}$ & $\begin{array}{l}\text { CD68 } \\
\text { CD68 }\end{array}$ & $\begin{array}{l}39 \% \\
36 \%\end{array}$ \\
\hline Vaysse (2017) [83] & FFPE tumor tissue & Unknown & CD68 & $54 \%$ \\
\hline Brown (2017) [72] & FFPE non-tumor tissue & 5 & CD68 & $57 \%$ \\
\hline Iyengar (2015) [73] & FFPE non-tumor tissue & 5 & CD68 & $51 \%$ \\
\hline Morris (2011) [67] & FFPE non-tumor tissue & $4-5$ & CD68 & $47 \%$ \\
\hline
\end{tabular}

Abbreviations: AA = African American; BBD = benign breast disease; $\mathrm{CA}=$ Caucasian; CLS-B = crown-like structures in breast adipose tissue; FFPE = formalin-fixed paraffin-embedded; KTB = Komen Normal Tissue Bank; NBLA = non-Black Latina; US = United States.

The number of adipose tissue samples used for CLS-B evaluation influences how many participants are classified as having CLS-B present (assuming the number of specimens examined positively correlates with the total area of adipocytes examined). Using only one tissue specimen per participant results in a lower proportion with CLS-B detected, as demonstrated in the studies by Maliniak et al. [69] and Mullooly et al. [80] where 30\% and $36 \%$ of breast cancer patients were reported with CLS-B present. These proportions are lower than all of the studies that used 4-5 specimens per case, even the one study of ideal-weight women, where $39 \%$ of patients were classified with CLS-B [71]. It is likely that some subjects who truly have CLS-B are misclassified as not having CLS-B when using only one tissue specimen. In a small study of 30 patients, $14(47 \%)$ had CLS-B present on $\geq 1$ of 5 tissue slides examined per patient [67]. Of these 14 patients, seven had CLS-B present on a single slide, six had CLS-B present on 2-4 slides, and only one patient had CLS-B present on all slides [67]. This small study provides evidence that CLS-B is heterogeneous across breast adipose tissue samples; sensitivity might be low for detecting CLS-B by only using one sample. However, the cost, availability of tissue, and pathologist time spent assessing CLS-B on five specimens per participant might be prohibitive- especially for large-scale epidemiologic studies, which are needed to adequately assess the prognostic value of CLSB. High-throughput assessment of CLS-B using novel imaging technologies [92] would decrease pathologist time but have not been used for detection of CLS-B. To balance cost 
and resources with potential misclassification, additional data evaluating the number of tissue specimens required for adequate CLS-B assessment are needed.

The antibody used for detecting CLS-B has varied across studies, with some using multiple markers. Although most previous investigations used the CD68 pan-macrophage marker, two studies employing macrophage markers specific to M1 and M2 phenotypes found differential associations with patient and tumor characteristics [86] as well as prognosis [87]. It is unknown whether differences detected by macrophage markers are biologically or clinically meaningful as they could have been due to chance in these relatively small studies. However, given the biologic differences between pro-inflammatory M1 and anti-inflammatory M2 macrophages, they could affect tumor biology differently [7,93]. CLS are thought to primarily consist of pro-inflammatory M1 macrophages since they are associated with a pro-inflammatory microenvironment [94]; however, both M1 and M2 macrophages increase in the adipose tissue microenvironment with obesity and their role in the tumor microenvironment differs. Anti-inflammatory M2 macrophages are considered protumorigenic as they express immunosuppressive molecules such as IL-10, programmed death-ligand 1 (PD-L1), and transforming growth factor (TGF)- $\beta$-favoring tumor growth. In contrast, M1 macrophages are considered antitumorigenic as they express pro-inflammatory, microbicidal cytokines [93,94]. It has been proposed that the ratio of M1/M2 macrophages may be a more biologically relevant indicator for cancer prognosis [93]. It is unclear how this emerging evidence of the diversity of the immune cell landscape might impact studies of CLS-B.

Finally, most studies among breast cancer patients have examined CLS-B in adipose tissue remote from the tumor-although a few have examined them more proximal to the tumor $[83,86,87]$. Tumor tissue is more widely available, especially for early-stage breast cancer patients who may opt for breast-conserving surgery, so evaluation of CLS-B in the surrounding adipose tissue would allow for assessment in a wider population. However, differences in CLS-B by proximity to the tumor are unclear. One study by Cha et al. found that CLS-B were present in 13-18\% of tumor-containing tissue, but no CLS-B were detected in non-tumor-containing tissue [86]. Although this contrasts with several studies that utilized non-tumor-containing tissue from mastectomy and found up to $57 \%$ of patients with CLS-B $[69,71,73,80-82]$, it does support the possibility that CLS-B are more frequent in close proximity to the tumor. No study has directly compared CLS-B associations with patient characteristics or prognosis by proximity to the tumor within the same study population. However, a study by Vaysse et al. examined CLS-B in adipose tissue surrounding the tumor primarily from lumpectomy specimens and found a strong association between obesity and CLS-B that was similar to associations found in studies where CLS-B were evaluated in non-tumor-containing tissue [83]. Nevertheless, studies should carefully consider assessment methods and their implications. CLS-B remote from the tumor may be more representative of inflammation that existed prior to breast malignancy while CLS-B in close proximity to the tumor could be a direct result of the tumor or diagnostic work-up (i.e., biopsy).

\subsection{The Role of CLS-B in the Incidence of Breast Cancer}

Assessing the role of CLS-B in breast cancer incidence is difficult, given that breast adipose tissue is not easily obtained or routinely collected. Obtaining tissue from normal breast tissue banks, reduction mammoplasty, or BBD cohorts provide opportunities to explore this association. Yet, substantial challenges remain. For instance, some studies suggest that the prevalence of CLS-B may be low among women without BBD or breast cancer (e.g., $3 \%$ and $18 \%$ in two samples of KTB normal breast tissue donors [84,85] and $2 \%$ in a sample of women who received reduction mammoplasty [86]). Thus, large study samples would be needed to adequately power these analyses. The greater detection of CLS-B among African American KTB donors found in one study (18\% with CLS-B present) suggests that studies may be more feasible in this group [84]. Thus, far, no study has examined whether CLS-B are associated with the risk of certain molecular subtypes of 
breast cancer. ER-negative breast cancers, including TNBC, are more frequent in African American women and are thought to be related to activation of NF- $\kappa B$ and inflammatory pathways [76,95]. The potentially higher frequency of CLS-B among African American women, who also tend to have a higher BMI than their European ancestry counterparts, could indicate a biologic explanation for the observed association between obesity and TNBC [17]. This hypothesis has not been tested.

The current studies of CLS-B and breast cancer risk were conducted within BBD cohorts, which is an opportune study group given that CLS-B is more frequent among BBD patients (compared to women without BBD) and breast adipose tissue from biopsy is more easily accessible. Although results from these studies may not be generalizable to women without BBD, they could provide some biologic insight-especially if future studies explore differences by menopausal status and molecular subtype. There may also be important public health and clinical implications for this select group. It has been suggested that CLS-B could be used to identify BBD patients exhibiting metabolic obesity who might benefit from behavioral changes or more frequent breast cancer screening [84]. Prevention measures based on CLS-B status would not be practical outside of this group unless a less invasive biomarker associated with CLS-B is identified.

\subsection{The Role of CLS-B in Predicting Therapeutic Effectiveness and Breast Cancer Prognosis}

Since breast tissue is often obtained from breast cancer patients receiving surgery as part of their treatment, investigating the association between CLS-B and prognosis is more feasible and likely to have greater public health implications than uncovering any etiologic association. Based on current evidence, the role of CLS-B in breast cancer prognosis is unclear. Well-designed and well-powered studies are needed to determine the predictive and prognostic value of CLS-B.

Future studies should examine heterogeneity in associations by host and tumor characteristics as well as in treatments received. Given the proposed mechanisms of CLS-B and clinical evidence that obesity affects the pharmacokinetics and effectiveness of some chemo- and hormonal therapies [96-103], these lesions may affect certain subgroups of breast cancer patients more than others [1,104]. Adipocyte-tumor crosstalk is an active area of preclinical research, with some studies in breast and other cancers showing that adipocytes can promote chemoresistance through various mechanisms $[105,106]$. Moreover, macrophages may further protect tumor cells from the cytotoxic effects of chemotherapyas suggested in several in vitro studies [7,107,108]. Thus, adipocytes and macrophages, such as those in CLS-B, might work together to reduce efficacy of cancer therapies. Future preclinical and clinical studies should further explore these potential interactions.

Given that CLS-B are associated with increased aromatase expression and activity, as supported by several animal and human studies $[67,71,73,79,80]$, they may play a role in the efficacy of breast cancer therapies that target aromatase. While the evidence is mixed, some studies have shown aromatase inhibitors (AIs) but not tamoxifen (which targets the estrogen receptor directly) to be less effective among postmenopausal women with a high BMI [109]. The decreased efficacy is thought to be due to increased aromatization from adipose tissue of androstenedione to estrogens among overweight/obese women, resulting in insufficient suppression by standard treatment doses [33,109]. CLS-B detection and density might be a better marker of increased aromatization than BMI as a considerable proportion of ideal-weight women have CLS-B [71], and CLS-B are associated with increased aromatization in breast fat [80]. How CLS-B may influence the effectiveness of AIs has yet to be investigated.

The inflammatory potential of breast adipose tissue will be insufficiently quantified based on the presence or absence of CLS-B. Instead, focusing on CLS-B density may be one option for better determining the prognostic value of CLS-B. Combining indicators of inflammation such as evidence of local breast adipose tissue inflammation (as indicated by CLS-B and NF- $\mathrm{KB}$ binding activity in the breast tissue) and systemic inflammation (as indicated by elevated cytokines and chemokines in the blood) may be another way to identify 
women at particularly high risk of poor breast cancer outcomes. If so, these patients might benefit from lifestyle modification such as increased physical activity or treatment with pharmacologic agents such as non-steroidal anti-inflammatory drugs (NSAIDs), metformin, or statins that target obesity-related conditions and inflammation [110-112]. Findings from a recent preclinical rat model of ER-positive postmenopausal breast cancer showed that treatment with metformin decreased the size of existing mammary tumors and saw improvements in mammary tissue inflammation by decreases in CLS [113]. Additionally, these drugs target some of the same inflammatory pathways as CLS-B. For instance, certain statins are associated with deactivating NF- $\mathrm{kB}$ activity, which may sensitize tumor cells to chemotherapy and prevent chemoresistance $[114,115]$. There is currently no epidemiologic data that have examined the combined effects of local and systemic inflammation on breast cancer outcomes or whether pharmacologic agents influence the effects of CLS-B on breast cancer outcomes. However, randomized controlled trials assessing the effects of statins (e.g., NCT04601116), NSAIDs (e.g., NCT02927249), and metformin (e.g., NCT01101438), as well as lifestyle changes (e.g., NCT02786875) on breast cancer prognosis are already underway (ClinicalTrials.gov. Available online: www.clinicaltrials.gov) (accessed on 28 April 2021).

\section{Conclusions}

Obesity increases the incidence of postmenopausal HR+ tumors (and possibly premenopausal TNBC), and worsens prognosis, with limited evidence of differences by menopausal or HR status. The underlying mechanisms for these associations are not fully understood, but growing evidence points to the adipose tissue microenvironment playing an important role given the prevalence of adipose tissue in the breast and its metabolic and inflammatory activity. CLS-B are inflammatory lesions within the breast adipose tissue microenvironment associated with local NF- $\mathrm{kB}$ activation, increased aromatase activity, and elevation of pro-inflammatory mediators-all which could have numerous effects on breast cancer development and progression. While the evidence is continuing to accumulate, there is currently not enough epidemiologic data to answer key questions about the role of CLS-B in breast cancer etiology or prognosis overall or by molecular subtype. Interpretation of this evidence base is further complicated by small sample sizes, a lack of standardized methodology in CLS-B assessment, and other challenges such as tissue collection for assessment. Future epidemiologic studies should take these into consideration.

Author Contributions: M.L.M., J.M.-K., D.P.C.-F., T.L.L., K.G., E.A.M.J., and L.E.M.: conceptualization and critically reviewing the article for important intellectual content; M.L.M.: writing-original draft; M.L.M., J.M.-K., and L.E.M.: literature review, writing-review and editing; L.E.M.: supervision. All authors have read and agreed to the published version of the manuscript.

Funding: This research received no external funding.

Institutional Review Board Statement: Not applicable.

Data Availability Statement: No new data were generated or analyzed in support of this research.

Conflicts of Interest: Gogineni reports receiving research funding from Genetech, Merck, Novartis, Pfizer, and Seagen for her role at Winship Cancer Institute/Emory University as site principal investigator, a one-time role on the advisory board to Pfizer, and receiving honoraria for participating in an OncLive presentation. No potential conflicts of interest were disclosed by the other authors.

\section{References}

1. Jiralerspong, S.; Goodwin, P.J. Obesity and Breast Cancer Prognosis: Evidence, Challenges, and Opportunities. J. Clin. Oncol. 2016, 34, 4203-4216. [CrossRef]

2. Lauby-Secretan, B.; Scoccianti, C.; Loomis, D.; Grosse, Y.; Bianchini, F.; Straif, K.; International Agency for Research on Cancer Handbook Working Group. Body Fatness and Cancer-Viewpoint of the IARC Working Group. N. Engl. J. Med. 2016, 375, 794-798. [CrossRef]

3. Chan, D.S.; Vieira, A.R.; Aune, D.; Bandera, E.V.; Greenwood, D.C.; McTiernan, A.; Navarro Rosenblatt, D.; Thune, I.; Vieira, R.; Norat, T. Body mass index and survival in women with breast cancer-systematic literature review and meta-analysis of 82 follow-up studies. Ann. Oncol. 2014, 25, 1901-1914. [CrossRef] 
4. Protani, M.; Coory, M.; Martin, J.H. Effect of obesity on survival of women with breast cancer: Systematic review and meta-analysis. Breast Cancer Res. Treat. 2010, 123, 627-635. [CrossRef]

5. Thun, M.J.; Linet, M.S.; Cerhan, J.R.; Haiman, C.; Schottenfeld, D. Schottenfeld and Fraumeni Cancer Epidemiology and Prevention, 4th ed.; Oxford University Press: New York, NY, USA, 2018.

6. Koshiol, J.; Lin, S.-W. Can tissue-based immune markers be used for studying the natural history of cancer? Ann. Epidemiol. 2012, 22, 520. [CrossRef] [PubMed]

7. Quail, D.F.; Dannenberg, A.J. The obese adipose tissue microenvironment in cancer development and progression. Nat. Rev. Endocrinol. 2019, 15, 139-154. [CrossRef]

8. Berger, N.A. Crown-like structures in breast adipose tissue from normal weight women: Important impact. Cancer Prev. Res. 2017. [CrossRef]

9. Suzuki, R.; Saji, S.; Toi, M. Impact of body mass index on breast cancer in accordance with the life-stage of women. Front. Oncol. 2012, 2, 123. [CrossRef]

10. James, F.; Wootton, S.; Jackson, A.; Wiseman, M.; Copson, E.; Cutress, R. Obesity in breast cancer-what is the risk factor? Eur. J. Cancer 2015, 51, 705-720. [CrossRef] [PubMed]

11. Chan, D.S.; Abar, L.; Cariolou, M.; Nanu, N.; Greenwood, D.C.; Bandera, E.V.; McTiernan, A.; Norat, T. World Cancer Research Fund International: Continuous Update Project-Systematic literature review and meta-analysis of observational cohort studies on physical activity, sedentary behavior, adiposity, and weight change and breast cancer risk. Cancer Causes Control. 2019, 30, 1183-1200. [CrossRef]

12. Munsell, M.F.; Sprague, B.L.; Berry, D.A.; Chisholm, G.; Trentham-Dietz, A. Body mass index and breast cancer risk according to postmenopausal estrogen-progestin use and hormone receptor status. Epidemiol. Rev. 2014, 36, 114-136. [CrossRef]

13. Premenopausal Breast Cancer Collaborative Group. Association of Body Mass Index and Age with Subsequent Breast Cancer Risk in Premenopausal Women. JAMA Oncol. 2018, 4, e181771. [CrossRef] [PubMed]

14. Yang, X.R.; Chang-Claude, J.; Goode, E.L.; Couch, F.J.; Nevanlinna, H.; Milne, R.L.; Gaudet, M.; Schmidt, M.K.; Broeks, A.; Cox, A.; et al. Associations of breast cancer risk factors with tumor subtypes: A pooled analysis from the Breast Cancer Association Consortium studies. J. Natl. Cancer Inst. 2011, 103, 250-263. [CrossRef] [PubMed]

15. Bandera, E.V.; Chandran, U.; Hong, C.-C.; Troester, M.A.; Bethea, T.N.; Adams-Campbell, L.L.; Haiman, C.A.; Park, S.-Y.; Olshan, A.F.; Ambrosone, C.B. Obesity, body fat distribution, and risk of breast cancer subtypes in African American women participating in the AMBER Consortium. Breast Cancer Res. Treat. 2015, 150, 655-666. [CrossRef]

16. Ma, H.; Ursin, G.; Xu, X.; Lee, E.; Togawa, K.; Malone, K.E.; Marchbanks, P.A.; McDonald, J.A.; Simon, M.S.; Folger, S.G. Body mass index at age 18 years and recent body mass index in relation to risk of breast cancer overall and ER/PR/HER2-defined subtypes in white women and African-American women: A pooled analysis. Breast Cancer Res. 2018, 20, 5. [CrossRef]

17. Pierobon, M.; Frankenfeld, C.L. Obesity as a risk factor for triple-negative breast cancers: A systematic review and meta-analysis. Breast Cancer Res. Treat. 2013, 137, 307-314. [CrossRef]

18. Houghton, S.C.; Eliassen, H.; Tamimi, R.M.; Willett, W.C.; Rosner, B.A.; Hankinson, S.E. Central adiposity and subsequent risk of breast cancer by menopause status. J. Natl. Cancer Inst. 2020. [CrossRef]

19. White, A.J.; Nichols, H.B.; Bradshaw, P.T.; Sandler, D.P. Overall and central adiposity and breast cancer risk in the Sister Study. Cancer 2015, 121, 3700-3708. [CrossRef]

20. Fagherazzi, G.; Chabbert-Buffet, N.; Fabre, A.; Guillas, G.; Boutron-Ruault, M.C.; Mesrine, S.; Clavel-Chapelon, F. Hip circumference is associated with the risk of premenopausal ER-/PR- breast cancer. Int. J. Obes. 2012, 36, 431-439. [CrossRef]

21. Canchola, A.J.; Anton-Culver, H.; Bernstein, L.; Clarke, C.A.; Henderson, K.; Ma, H.; Ursin, G.; Horn-Ross, P.L. Body size and the risk of postmenopausal breast cancer subtypes in the California Teachers Study cohort. Cancer Causes Control. 2012. [CrossRef] [PubMed]

22. Deng, T.; Lyon, C.J.; Bergin, S.; Caligiuri, M.A.; Hsueh, W.A. Obesity, Inflammation, and Cancer. Annu. Rev. Pathol. 2016, 11, 421-449. [CrossRef] [PubMed]

23. Iyengar, N.M.; Hudis, C.A.; Dannenberg, A.J. Obesity and inflammation: New insights into breast cancer development and progression. Am. Soc. Clin. Oncol. Educ. Book 2013, 33, 46-51. [CrossRef]

24. Poloz, Y.; Stambolic, V. Obesity and cancer, a case for insulin signaling. Cell Death Dis. 2015, 6, e2037. [CrossRef]

25. Agurs-Collins, T.; Ross, S.A.; Dunn, B.K. The Many Faces of Obesity and Its Influence on Breast Cancer Risk. Front. Oncol. 2019, 9, 765. [CrossRef]

26. Shuster, A.; Patlas, M.; Pinthus, J.H.; Mourtzakis, M. The clinical importance of visceral adiposity: A critical review of methods for visceral adipose tissue analysis. Br. J. Radiol. 2012, 85, 1-10. [CrossRef] [PubMed]

27. Ibrahim, M.M. Subcutaneous and visceral adipose tissue: Structural and functional differences. Obes. Rev. 2010, 11, 11-18. [CrossRef] [PubMed]

28. Niraula, S.; Ocana, A.; Ennis, M.; Goodwin, P.J. Body size and breast cancer prognosis in relation to hormone receptor and menopausal status: A meta-analysis. Breast Cancer Res. Treat. 2012, 134, 769-781. [CrossRef]

29. Ewertz, M.; Gray, K.P.; Regan, M.M.; Ejlertsen, B.; Price, K.N.; Thurlimann, B.; Bonnefoi, H.; Forbes, J.F.; Paridaens, R.J.; Rabaglio, M.; et al. Obesity and risk of recurrence or death after adjuvant endocrine therapy with letrozole or tamoxifen in the breast international group 1-98 trial. J. Clin. Oncol. 2012, 30, 3967-3975. [CrossRef] 
30. Ewertz, M.; Jensen, M.B.; Gunnarsdottir, K.A.; Hojris, I.; Jakobsen, E.H.; Nielsen, D.; Stenbygaard, L.E.; Tange, U.B.; Cold, S. Effect of obesity on prognosis after early-stage breast cancer. J. Clin. Oncol. 2011, 29, 25-31. [CrossRef]

31. Goodwin, P.J.; Ennis, M.; Pritchard, K.I.; Trudeau, M.E.; Koo, J.; Taylor, S.K.; Hood, N. Insulin- and obesity-related variables in early-stage breast cancer: Correlations and time course of prognostic associations. J. Clin. Oncol. 2012, 30, 164-171. [CrossRef]

32. Nechuta, S.; Chen, W.Y.; Cai, H.; Poole, E.M.; Kwan, M.L.; Flatt, S.W.; Patterson, R.E.; Pierce, J.P.; Caan, B.J.; Ou Shu, X. A pooled analysis of post-diagnosis lifestyle factors in association with late estrogen-receptor-positive breast cancer prognosis. Int. J. Cancer 2016, 138, 2088-2097. [CrossRef] [PubMed]

33. Sestak, I.; Distler, W.; Forbes, J.F.; Dowsett, M.; Howell, A.; Cuzick, J. Effect of body mass index on recurrences in tamoxifen and anastrozole treated women: An exploratory analysis from the ATAC trial. J. Clin. Oncol. 2010, 28, 3411-3415. [CrossRef] [PubMed]

34. Norat, T.; Chan, D.; Vieira, A.R.; Aune, D.; Rosenblatt, D.N.; Vingeliene, S.; Abar, L.; Vieira, R. Systematic review on diet, nutrition, physical activity and survival and second cancers in breast cancer survivors. Contin. Update Proj. 2014, 222. Available online: https:/ / www.aicr.org/wp-content/uploads/2020/01/breast-cancer-survivors-slr.pdf (accessed on 28 April 2021).

35. Mei, L.; He, L.; Song, Y.; Lv, Y.; Zhang, L.; Hao, F.; Xu, M. Association between obesity with disease-free survival and overall survival in triple-negative breast cancer: A meta-analysis. Medicine 2018, 97, e0719. [CrossRef]

36. Iyengar, N.M.; Gucalp, A.; Dannenberg, A.J.; Hudis, C.A. Obesity and cancer mechanisms: Tumor microenvironment and inflammation. J. Clin. Oncol. 2016, 34, 4270. [CrossRef]

37. Picon-Ruiz, M.; Morata-Tarifa, C.; Valle-Goffin, J.J.; Friedman, E.R.; Slingerland, J.M. Obesity and adverse breast cancer risk and outcome: Mechanistic insights and strategies for intervention. CA Cancer J. Clin. 2017, 67, 378-397. [CrossRef]

38. Park, J.; Morley, T.S.; Kim, M.; Clegg, D.J.; Scherer, P.E. Obesity and cancer-mechanisms underlying tumour progression and recurrence. Nat. Rev. Endocrinol. 2014, 10, 455-465. [CrossRef]

39. Lohmann, A.E.; Goodwin, P.J.; Chlebowski, R.T.; Pan, K.; Stambolic, V.; Dowling, R.J. Association of Obesity-Related Metabolic Disruptions with Cancer Risk and Outcome. J. Clin. Oncol. 2016, 34, 4249-4255. [CrossRef]

40. Zwick, R.K.; Guerrero-Juarez, C.F.; Horsley, V.; Plikus, M.V. Anatomical, physiological, and functional diversity of adipose tissue. Cell Metab. 2018, 27, 68-83. [CrossRef]

41. Kothari, C.; Diorio, C.; Durocher, F. The Importance of Breast Adipose Tissue in Breast Cancer. Int. J. Mol. Sci. 2020, 27, 5760. [CrossRef]

42. Key, T.J.; Appleby, P.N.; Reeves, G.K.; Roddam, A.; Dorgan, J.F.; Longcope, C.; Stanczyk, F.Z.; Stephenson, H.E., Jr.; Falk, R.T.; Miller, R.; et al. Body mass index, serum sex hormones, and breast cancer risk in postmenopausal women. J. Natl. Cancer Inst. 2003, 95, 1218-1226. [CrossRef]

43. Dashti, S.G.; Simpson, J.A.; Karahalios, A.; Viallon, V.; Moreno-Betancur, M.; Gurrin, L.C.; MacInnis, R.J.; Lynch, B.M.; Baglietto, L.; Morris, H.A. Adiposity and estrogen receptor-positive, postmenopausal breast cancer risk: Quantification of the mediating effects of fasting insulin and free estradiol. Int. J. Cancer 2020, 146, 1541-1552. [CrossRef]

44. Hvidtfeldt, U.A.; Gunter, M.J.; Lange, T.; Chlebowski, R.T.; Lane, D.; Farhat, G.N.; Freiberg, M.S.; Keiding, N.; Lee, J.S.; Prentice, R.; et al. Quantifying mediating effects of endogenous estrogen and insulin in the relation between obesity, alcohol consumption, and breast cancer. Cancer Epidemiol. Biomark. Prev. 2012, 21, 1203-1212. [CrossRef]

45. Chen, Y.; Liu, L.; Zhou, Q.; Imam, M.U.; Cai, J.; Wang, Y.; Qi, M.; Sun, P.; Ping, Z.; Fu, X. Body mass index had different effects on premenopausal and postmenopausal breast cancer risks: A dose-response meta-analysis with 3,318,796 subjects from 31 cohort studies. BMC Public Health 2017, 17, 936. [CrossRef] [PubMed]

46. Castoldi, A.; Naffah de Souza, C.; Câmara, N.O.S.; Moraes-Vieira, P.M. The macrophage switch in obesity development. Front. Immunol. 2016, 6, 637. [CrossRef]

47. Bigornia, S.J.; Farb, M.G.; Mott, M.M.; Hess, D.T.; Carmine, B.; Fiscale, A.; Joseph, L.; Apovian, C.M.; Gokce, N. Relation of depot-specific adipose inflammation to insulin resistance in human obesity. Nutr. Diabetes 2012, 2, e30. [CrossRef] [PubMed]

48. Le, K.A.; Mahurkar, S.; Alderete, T.L.; Hasson, R.E.; Adam, T.C.; Kim, J.S.; Beale, E.; Xie, C.; Greenberg, A.S.; Allayee, H.; et al. Subcutaneous adipose tissue macrophage infiltration is associated with hepatic and visceral fat deposition, hyperinsulinemia, and stimulation of NF-kappaB stress pathway. Diabetes 2011, 60, 2802-2809. [CrossRef]

49. Wang, Y.Y.; Lehuede, C.; Laurent, V.; Dirat, B.; Dauvillier, S.; Bochet, L.; Le Gonidec, S.; Escourrou, G.; Valet, P.; Muller, C. Adipose tissue and breast epithelial cells: A dangerous dynamic duo in breast cancer. Cancer Lett. 2012, 324, 142-151. [CrossRef]

50. Zhao, X.B.; Ren, G.S. Diabetes mellitus and prognosis in women with breast cancer: A systematic review and meta-analysis Medicine 2016, 95, e5602. [CrossRef]

51. Larsson, S.C.; Mantzoros, C.S.; Wolk, A. Diabetes mellitus and risk of breast cancer: A meta-analysis. Int. J. Cancer 2007, 121, 856-862. [PubMed]

52. Bhandari, R.; Kelley, G.A.; Hartley, T.A.; Rockett, I.R. Metabolic syndrome is associated with increased breast cancer risk: A systematic review with meta-analysis. Int. J. Breast Cancer 2014, 2014. [CrossRef]

53. Ando, S.; Gelsomino, L.; Panza, S.; Giordano, C.; Bonofiglio, D.; Barone, I.; Catalano, S. Obesity, Leptin and Breast Cancer: Epidemiological Evidence and Proposed Mechanisms. Cancers 2019, 11, 62. [CrossRef]

54. Ye, J.; Jia, J.; Dong, S.; Zhang, C.; Yu, S.; Li, L.; Mao, C.; Wang, D.; Chen, J.; Yuan, G. Circulating adiponectin levels and the risk of breast cancer: A meta-analysis. Eur. J. Cancer Prev. 2014, 23, 158-165. [CrossRef] 
55. Dimou, N.L.; Papadimitriou, N.; Gill, D.; Christakoudi, S.; Murphy, N.; Gunter, M.J.; Travis, R.C.; Key, T.J.; Fortner, R.T.; Haycock, P.C. Sex hormone binding globulin and risk of breast cancer: A Mendelian randomization study. Int. J. Epidemiol. 2019, 48, 807-816. [CrossRef] [PubMed]

56. Renehan, A.G.; Zwahlen, M.; Minder, C.; O’Dwyer, S.T.; Shalet, S.M.; Egger, M. Insulin-like growth factor (IGF)-I, IGF binding protein-3, and cancer risk: Systematic review and meta-regression analysis. Lancet 2004, 363, 1346-1353. [CrossRef]

57. Christopoulos, P.F.; Msaouel, P.; Koutsilieris, M. The role of the insulin-like growth factor-1 system in breast cancer. Mol. Cancer 2015, 14, 43. [CrossRef]

58. Renehan, A.G.; Harvie, M.; Howell, A. Insulin-like growth factor (IGF)-I, IGF binding protein-3, and breast cancer risk: Eight years on. Endocr. Relat. Cancer 2006, 13, 273-278. [CrossRef] [PubMed]

59. The Endogenous Hormones Breast Cancer Collaborative Group. Insulin-like growth factor 1 (IGF1), IGF binding protein 3 (IGFBP3), and breast cancer risk: Pooled individual data analysis of 17 prospective studies. Lancet Oncol. 2010, 11, 530-542. [CrossRef]

60. Autier, P.; Koechlin, A.; Boniol, M.; Mullie, P.; Bolli, G.; Rosenstock, J.; Boyle, P. Serum insulin and C-peptide concentration and breast cancer: A meta-analysis. Cancer Causes Control. 2013, 24, 873-883. [CrossRef]

61. Guo, L.; Liu, S.; Zhang, S.; Chen, Q.; Zhang, M.; Quan, P.; Lu, J.; Sun, X. C-reactive protein and risk of breast cancer: A systematic review and meta-analysis. Sci. Rep. 2015, 5, 10508. [CrossRef]

62. Kehm, R.D.; McDonald, J.A.; Fenton, S.E.; Kavanaugh-Lynch, M.; Leung, K.A.; McKenzie, K.E.; Mandelblatt, J.S.; Terry, M.B Inflammatory Biomarkers and Breast Cancer Risk: A Systematic Review of the Evidence and Future Potential for Intervention Research. Int. J. Environ. Res. Public Health 2020, 17, 5445. [CrossRef]

63. Pettersson, A.; Tamimi, R.M. Breast fat and breast cancer. Breast Cancer Res. Treat. 2012, 135, 321-323. [CrossRef]

64. Dowsett, M.; Folkerd, E. Reduced progesterone levels explain the reduced risk of breast cancer in obese premenopausal women: A new hypothesis. Breast Cancer Res. Treat. 2015, 149, 1-4. [CrossRef] [PubMed]

65. Terry, M.B.; Cohn, B.A.; Goldberg, M.; Flom, J.D.; Wei, Y.; Houghton, L.C.; Tehranifar, P.; McDonald, J.A.; Protacio, A.; Cirillo, P.; et al. Do Birth Weight and Weight Gain During Infancy and Early Childhood Explain Variation in Mammographic Density in Women in Midlife? Results From Cohort and Sibling Analyses. Am. J. Epidemiol. 2019, 188, 294-304. [CrossRef]

66. Rice, M.S.; Bertrand, K.A.; VanderWeele, T.J.; Rosner, B.A.; Liao, X.; Adami, H.-O.; Tamimi, R.M. Mammographic density and breast cancer risk: A mediation analysis. Breast Cancer Res. 2016, 18, 1-13. [CrossRef]

67. Morris, P.G.; Hudis, C.A.; Giri, D.; Morrow, M.; Falcone, D.J.; Zhou, X.K.; Du, B.; Brogi, E.; Crawford, C.B.; Kopelovich, L.; et al. Inflammation and increased aromatase expression occur in the breast tissue of obese women with breast cancer. Cancer Prev. Res. 2011, 4, 1021-1029. [CrossRef]

68. Cinti, S.; Mitchell, G.; Barbatelli, G.; Murano, I.; Ceresi, E.; Faloia, E.; Wang, S.; Fortier, M.; Greenberg, A.S.; Obin, M.S. Adipocyte death defines macrophage localization and function in adipose tissue of obese mice and humans. J. Lipid Res. 2005, 46, $2347-2355$. [CrossRef]

69. Maliniak, M.L.; Cheriyan, A.M.; Sherman, M.E.; Liu, Y.; Gogineni, K.; Liu, J.; He, J.; Krishnamurti, U.; Miller-Kleinhenz, J.; Ashiqueali, R.; et al. Detection of crown-like structures in breast adipose tissue and clinical outcomes among African-American and White women with breast cancer. Breast Cancer Res. 2020, 22, 65. [CrossRef]

70. Greenlee, H.; Shi, Z.; Hibshoosh, H.; Giri, D.D.; Ahmed, A.; Williams, S.; Falcone, D.J.; Winston, L.A.; Zhou, X.K.; Hudis, C.A.; et al. Obesity-associated Breast Inflammation among Hispanic/Latina Breast Cancer Patients. Cancer Prev. Res. 2019, 12, 21-30. [CrossRef]

71. Iyengar, N.M.; Brown, K.A.; Zhou, X.K.; Gucalp, A.; Subbaramaiah, K.; Giri, D.D.; Zahid, H.; Bhardwaj, P.; Wendel, N.K.; Falcone, D.J.; et al. Metabolic Obesity, Adipose Inflammation and Elevated Breast Aromatase in Women with Normal Body Mass Index. Cancer Prev. Res. 2017, 10, 235-243. [CrossRef]

72. Brown, K.A.; Iyengar, N.M.; Zhou, X.K.; Gucalp, A.; Subbaramaiah, K.; Wang, H.; Giri, D.D.; Morrow, M.; Falcone, D.J.; Wendel, N.K. Menopause is a determinant of breast aromatase expression and its associations with BMI, inflammation, and systemic markers. J. Clin. Endocrinol. Metab. 2017, 102, 1692-1701. [CrossRef]

73. Iyengar, N.M.; Morris, P.G.; Zhou, X.K.; Gucalp, A.; Giri, D.; Harbus, M.D.; Falcone, D.J.; Krasne, M.D.; Vahdat, L.T.; Subbaramaiah, K.; et al. Menopause is a determinant of breast adipose inflammation. Cancer Prev. Res. 2015, 8, 349-358. [CrossRef] [PubMed]

74. Iyengar, N.M.; Hudis, C.A.; Dannenberg, A.J. Obesity and cancer: Local and systemic mechanisms. Annu. Rev. Med. 2015, 66, 297-309. [CrossRef] [PubMed]

75. Howe, L.R.; Subbaramaiah, K.; Hudis, C.A.; Dannenberg, A.J. Molecular pathways: Adipose inflammation as a mediator of obesity-associated cancer. Clin. Cancer Res. 2013, 19, 6074-6083. [CrossRef]

76. Wang, W.; Nag, S.A.; Zhang, R. Targeting the NFkappaB signaling pathways for breast cancer prevention and therapy. Curr. Med. Chem. 2015, 22, 264-289. [CrossRef]

77. Rose, D.P.; Vona-Davis, L. Biochemical and molecular mechanisms for the association between obesity, chronic inflammation, and breast cancer. Biofactors 2014, 40, 1-12. [CrossRef]

78. Engin, A.B.; Engin, A.; Gonul, I.I. The effect of adipocyte-macrophage crosstalk in obesity-related breast cancer. J. Mol. Endocrinol. 2019, 62, R201-R222. [CrossRef] 
79. Subbaramaiah, K.; Howe, L.R.; Bhardwaj, P.; Du, B.; Gravaghi, C.; Yantiss, R.K.; Zhou, X.K.; Blaho, V.A.; Hla, T.; Yang, P.; et al. Obesity is associated with inflammation and elevated aromatase expression in the mouse mammary gland. Cancer Prev. Res. 2011, 4, 329-346. [CrossRef] [PubMed]

80. Mullooly, M.; Yang, H.P.; Falk, R.T.; Nyante, S.J.; Cora, R.; Pfeiffer, R.M.; Radisky, D.C.; Visscher, D.W.; Hartmann, L.C.; Carter, J.M.; et al. Relationship between crown-like structures and sex-steroid hormones in breast adipose tissue and serum among postmenopausal breast cancer patients. Breast Cancer Res. 2017, 19, 8. [CrossRef]

81. Iyengar, N.M.; Chen, I.C.; Zhou, X.K.; Giri, D.D.; Falcone, D.J.; Winston, L.A.; Wang, H.; Williams, S.; Lu, Y.S.; Hsueh, T.H.; et al. Adiposity, Inflammation, and Breast Cancer Pathogenesis in Asian Women. Cancer Prev. Res. 2018, 11, 227-236. [CrossRef]

82. Iyengar, N.M.; Zhou, X.K.; Gucalp, A.; Morris, P.G.; Howe, L.R.; Giri, D.D.; Morrow, M.; Wang, H.; Pollak, M.; Jones, L.W.; et al. Systemic Correlates of White Adipose Tissue Inflammation in Early-Stage Breast Cancer. Clin. Cancer Res. 2016, 22, 2283-2289. [CrossRef]

83. Vaysse, C.; Lomo, J.; Garred, O.; Fjeldheim, F.; Lofteroed, T.; Schlichting, E.; McTiernan, A.; Frydenberg, H.; Husoy, A.; Lundgren, S.; et al. Inflammation of mammary adipose tissue occurs in overweight and obese patients exhibiting early-stage breast cancer. NPJ Breast Cancer 2017, 3, 19. [CrossRef]

84. Shaik, A.N.; Kiavash, K.; Stark, K.; Boerner, J.L.; Ruterbusch, J.J.; Deirawan, H.; Bandyopadhyay, S.; Ali-Fehmi, R.; Dyson, G.; Cote, M.L. Inflammation markers on benign breast biopsy are associated with risk of invasive breast cancer in African American women. Breast Cancer Res. Treat. 2020, 185, 1-9. [CrossRef] [PubMed]

85. Carter, J.M.; Hoskin, T.L.; Pena, M.A.; Brahmbhatt, R.; Winham, S.J.; Frost, M.H.; Stallings-Mann, M.; Radisky, D.C.; Knutson, K.L.; Visscher, D.W.; et al. Macrophagic "Crown-like Structures" Are Associated with an Increased Risk of Breast Cancer in Benign Breast Disease. Cancer Prev. Res. 2018, 11, 113-119. [CrossRef]

86. Cha, Y.J.; Kim, E.S.; Koo, J.S. Tumor-associated macrophages and crown-like structures in adipose tissue in breast cancer. Breast Cancer Res. Treat. 2018, 170, 15-25. [CrossRef]

87. Koru-Sengul, T.; Santander, A.M.; Miao, F.; Sanchez, L.G.; Jorda, M.; Gluck, S.; Ince, T.A.; Nadji, M.; Chen, Z.; Penichet, M.L.; et al. Breast cancers from black women exhibit higher numbers of immunosuppressive macrophages with proliferative activity and of crown-like structures associated with lower survival compared to non-black Latinas and Caucasians. Breast Cancer Res. Treat. 2016, 158, 113-126. [CrossRef]

88. Kelemen, L.E.; Pankratz, V.S.; Sellers, T.A.; Brandt, K.R.; Wang, A.; Janney, C.; Fredericksen, Z.S.; Cerhan, J.R.; Vachon, C.M. Age-specific trends in mammographic density: The Minnesota Breast Cancer Family Study. Am. J. Epidemiol. 2008, 167, 1027-1036. [CrossRef]

89. Mancuso, P.; Bouchard, B. The impact of aging on adipose function and adipokine synthesis. Front. Endocrinol. 2019, 10, 137. [CrossRef]

90. Cossrow, N.; Falkner, B. Race/ethnic issues in obesity and obesity-related comorbidities. J. Clin. Endocrinol. Metab. 2004, 89, 2590-2594. [CrossRef]

91. Wilk, J.B.; Lash, T.L. Risk factor studies of age-at-onset in a sample ascertained for Parkinson disease affected sibling pairs: A cautionary tale. Emerg. Themes Epidemiol. 2007, 4, 1. [CrossRef]

92. Haka, A.S.; Sue, E.; Zhang, C.; Bhardwaj, P.; Sterling, J.; Carpenter, C.; Leonard, M.; Manzoor, M.; Walker, J.; Aleman, J.O.; et al. Noninvasive Detection of Inflammatory Changes in White Adipose Tissue by Label-Free Raman Spectroscopy. Anal. Chem. 2016, 88, 2140-2148. [CrossRef]

93. Jayasingam, S.D.; Citartan, M.; Thang, T.H.; Zin, A.A.M.; Ang, K.C.; Ch'ng, E.S. Evaluating the polarization of tumor-associated macrophages into M1 and M2 phenotypes in human cancer tissue: Technicalities and challenges in routine clinical practice. Front. Oncol. 2019, 9, 1512. [CrossRef]

94. Springer, N.L.; Iyengar, N.M.; Bareja, R.; Verma, A.; Jochelson, M.S.; Giri, D.D.; Zhou, X.K.; Elemento, O.; Dannenberg, A.J.; Fischbach, C. Obesity-associated extracellular matrix remodeling promotes a macrophage phenotype similar to tumor-associated macrophages. Am. J. Pathol. 2019, 189. [CrossRef]

95. Ossovskaya, V.; Wang, Y.; Budoff, A.; Xu, Q.; Lituev, A.; Potapova, O.; Vansant, G.; Monforte, J.; Daraselia, N. Exploring molecular pathways of triple-negative breast cancer. Genes Cancer 2011, 2, 870-879. [CrossRef]

96. Blouin, R.A.; Warren, G.W. Pharmacokinetic considerations in obesity. J. Pharm. Sci. 1999, 88, 1-7. [CrossRef]

97. de Azambuja, E.; McCaskill-Stevens, W.; Francis, P.; Quinaux, E.; Crown, J.P.; Vicente, M.; Giuliani, R.; Nordenskjold, B.; Gutierez, J.; Andersson, M.; et al. The effect of body mass index on overall and disease-free survival in node-positive breast cancer patients treated with docetaxel and doxorubicin-containing adjuvant chemotherapy: The experience of the BIG 02-98 trial. Breast Cancer Res. Treat. 2010, 119, 145-153. [CrossRef]

98. Desmedt, C.; Fornili, M.; Clatot, F.; Demicheli, R.; De Bortoli, D.; Di Leo, A.; Viale, G.; de Azambuja, E.; Crown, J.; Francis, P.A. Differential benefit of adjuvant docetaxel-based chemotherapy in patients with early breast cancer according to baseline body mass index. J. Clin. Oncol. 2020, 38, 2883-2891. [CrossRef]

99. Fontanella, C.; Lederer, B.; Gade, S.; Vanoppen, M.; Blohmer, J.U.; Costa, S.D.; Denkert, C.; Eidtmann, H.; Gerber, B.; Hanusch, C.; et al. Impact of body mass index on neoadjuvant treatment outcome: A pooled analysis of eight prospective neoadjuvant breast cancer trials. Breast Cancer Res. Treat. 2015, 150, 127-139. [CrossRef] 
100. Ladoire, S.; Dalban, C.; Roche, H.; Spielmann, M.; Fumoleau, P.; Levy, C.; Martin, A.L.; Ecarnot, F.; Bonnetain, F.; Ghiringhelli, F. Effect of obesity on disease-free and overall survival in node-positive breast cancer patients in a large French population: A pooled analysis of two randomised trials. Eur. J. Cancer 2014, 50, 506-516. [CrossRef]

101. Litton, J.K.; Gonzalez-Angulo, A.M.; Warneke, C.L.; Buzdar, A.U.; Kau, S.W.; Bondy, M.; Mahabir, S.; Hortobagyi, G.N.; Brewster, A.M. Relationship between obesity and pathologic response to neoadjuvant chemotherapy among women with operable breast cancer. J. Clin. Oncol. 2008, 26, 4072-4077. [CrossRef]

102. Pajares, B.; Pollan, M.; Martin, M.; Mackey, J.R.; Lluch, A.; Gavila, J.; Vogel, C.; Ruiz-Borrego, M.; Calvo, L.; Pienkowski, T.; et al. Obesity and survival in operable breast cancer patients treated with adjuvant anthracyclines and taxanes according to pathological subtypes: A pooled analysis. Breast Cancer Res. 2013, 15, R105. [CrossRef]

103. Ross, K.H.; Gogineni, K.; Subhedar, P.D.; Lin, J.Y.; McCullough, L.E. Obesity and cancer treatment efficacy: Existing challenges and opportunities. Cancer 2019, 125, 1588-1592. [CrossRef]

104. Zhao, C.; Wu, M.; Zeng, N.; Xiong, M.; Hu, W.; Lv, W.; Yi, Y.; Zhang, Q.; Wu, Y. Cancer-associated adipocytes: Emerging supporters in breast cancer. J. Exp. Clin. Cancer Res. 2020, 39, 156. [CrossRef] [PubMed]

105. Lehuede, C.; Li, X.; Dauvillier, S.; Vaysse, C.; Franchet, C.; Clement, E.; Esteve, D.; Longue, M.; Chaltiel, L.; Le Gonidec, S.; et al. Adipocytes promote breast cancer resistance to chemotherapy, a process amplified by obesity: Role of the major vault protein (MVP). Breast Cancer Res. 2019, 21, 7. [CrossRef]

106. Lengyel, E.; Makowski, L.; DiGiovanni, J.; Kolonin, M.G. Cancer as a Matter of Fat: The Crosstalk between Adipose Tissue and Tumors. Trends Cancer 2018, 4, 374-384. [CrossRef] [PubMed]

107. Olson, O.C.; Kim, H.; Quail, D.F.; Foley, E.A.; Joyce, J.A. Tumor-Associated Macrophages Suppress the Cytotoxic Activity of Antimitotic Agents. Cell Rep. 2017, 19, 101-113. [CrossRef] [PubMed]

108. Larionova, I.; Cherdyntseva, N.; Liu, T.; Patysheva, M.; Rakina, M.; Kzhyshkowska, J. Interaction of tumor-associated macrophages and cancer chemotherapy. Oncoimmunology 2019, 8, 1596004. [CrossRef]

109. Ioannides, S.J.; Barlow, P.L.; Elwood, J.M.; Porter, D. Effect of obesity on aromatase inhibitor efficacy in postmenopausal, hormone receptor-positive breast cancer: A systematic review. Breast Cancer Res. Treat. 2014, 147, 237-248. [CrossRef]

110. Kwan, M.L.; Habel, L.A.; Slattery, M.L.; Caan, B. NSAIDs and breast cancer recurrence in a prospective cohort study. Cancer Causes Control. 2007, 18, 613-620. [CrossRef]

111. Ahern, T.P.; Lash, T.L.; Damkier, P.; Christiansen, P.M.; Cronin-Fenton, D.P. Statins and breast cancer prognosis: Evidence and opportunities. Lancet Oncol. 2014, 15, e461-e468. [CrossRef]

112. Gong, Z.; Aragaki, A.K.; Chlebowski, R.T.; Manson, J.E.; Rohan, T.E.; Chen, C.; Vitolins, M.Z.; Tinker, L.F.; LeBlanc, E.S.; Kuller, L.H.; et al. Diabetes, metformin and incidence of and death from invasive cancer in postmenopausal women: Results from the women's health initiative. Int. J. Cancer 2016, 138, 1915-1927. [CrossRef]

113. Giles, E.D.; Jindal, S.; Wellberg, E.A.; Schedin, T.; Anderson, S.M.; Thor, A.D.; Edwards, D.P.; MacLean, P.S.; Schedin, P. Metformin inhibits stromal aromatase expression and tumor progression in a rodent model of postmenopausal breast cancer. Breast Cancer Res. 2018, 20, 50. [CrossRef]

114. Ahn, K.S.; Sethi, G.; Aggarwal, B.B. Reversal of chemoresistance and enhancement of apoptosis by statins through downregulation of the NF-кB pathway. Biochem. Pharmacol. 2008, 75, 907-913. [CrossRef]

115. Gazzerro, P.; Proto, M.C.; Gangemi, G.; Malfitano, A.M.; Ciaglia, E.; Pisanti, S.; Santoro, A.; Laezza, C.; Bifulco, M. Pharmacological actions of statins: A critical appraisal in the management of cancer. Pharmacol. Rev. 2012, 64, 102-146. [CrossRef] 